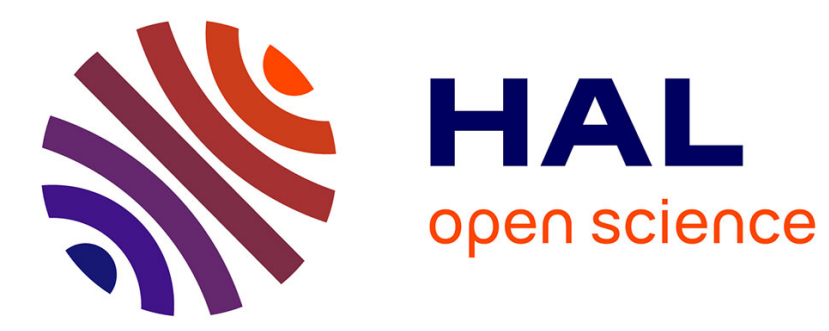

\title{
Transport of water and ions in partially water-saturated porous media. Part 3. Electrical conductivity
}

André Revil, Abdellahi Soueid Ahmed, Stephan Matthai

\section{To cite this version:}

André Revil, Abdellahi Soueid Ahmed, Stephan Matthai. Transport of water and ions in partially water-saturated porous media. Part 3. Electrical conductivity. Advances in Water Resources, 2018, 121, pp.97-111. 10.1016/j.advwatres.2018.08.007 . hal-02324271

\section{HAL Id: hal-02324271 \\ https://hal.science/hal-02324271}

Submitted on 23 Nov 2020

HAL is a multi-disciplinary open access archive for the deposit and dissemination of scientific research documents, whether they are published or not. The documents may come from teaching and research institutions in France or abroad, or from public or private research centers.
L'archive ouverte pluridisciplinaire HAL, est destinée au dépôt et à la diffusion de documents scientifiques de niveau recherche, publiés ou non, émanant des établissements d'enseignement et de recherche français ou étrangers, des laboratoires publics ou privés. 


\title{
Transport of water and ions in partially water-saturated porous media. Part 3. Electrical conductivity is
}

\author{
André Revil ${ }^{\mathrm{a}, *}$, Abdellahi Soueid Ahmed ${ }^{\mathrm{a}}$, Stephan Matthai ${ }^{\mathrm{b}}$ \\ ${ }^{a}$ Université Grenoble Alpes, Université Savoie Mont Blanc, CNRS, IRD, IFSTTAR, ISTerre, 38000 Grenoble, France \\ ${ }^{\mathrm{b}}$ Department of Infrastructure Engineering, University of Melbourne at Parkville, VIC 3010, Australia
}

\section{A B S T R A C T}

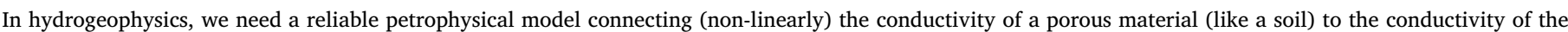

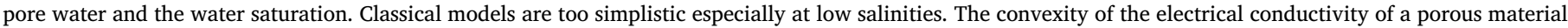

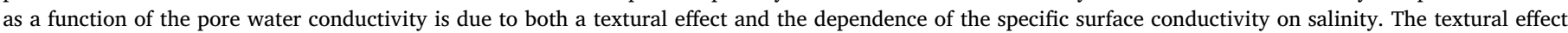

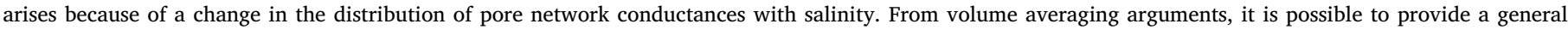

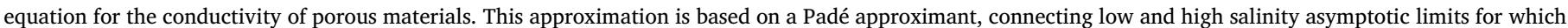

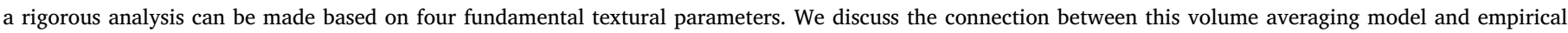

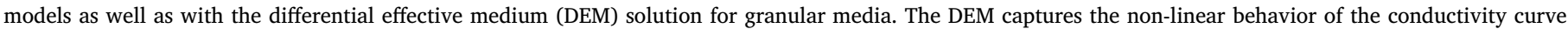

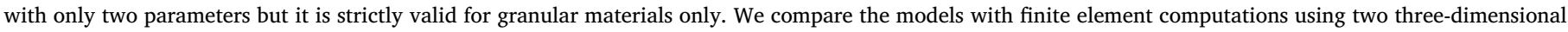
pore geometries with continuous and discontinuous solid surfaces, respectively. Finally the models are compared to experimental data.

\section{Introduction}

Seventy six years ago, Archie (1942) developed a simple equation to interpret resistivity well logs in clean (clay-free) formations. In his model, the electrical conductivity of a porous medium $\sigma$ (in $\mathrm{S} \mathrm{m}^{-1}$ ) is written as

$\sigma=\frac{1}{F} \sigma_{w}$

where $F=\phi^{-m}$ (dimensionless) describes the (resistivity) electrical formation factor, $\phi$ denotes the connected porosity, and $\sigma_{w}$ (in $\mathrm{S} \mathrm{m}^{-1}$ ) denotes the conductivity of the pore water, which depends in turn on the salinity of the pore water and temperature. The exponent $m$ (dimensionless) is called the cementation exponent in the petroleum engineering community. For a set of spherical particles, $m$ increases with cementation from 1.1-1.3 for colloidal suspensions and unconsolidated sands to 1.7-2.1 for consolidated sandstones where the grains are bonded together by cements (see Friedman, 2005). However, the value of $m$ also varies with grain shape (e.g., Jackson et al., 1978) and this terminology is therefore confusing. The term "Archie porosity exponent" (or first Archie exponent) should be preferred. Note that the power law relationship between electrical conductivity and porosity was already known much before Archie (1942), see for instance Bruggeman (1935). This version of Archie's law $F=\phi^{-m}$ should also not be confused with Archie's law written as $F=a \phi^{-m}$ (developed later in the $50 \mathrm{~s}$ ) and usually used to fit a formation factor / porosity data set in which each point corresponds to a distinct sample, all the sample being from the same facies (e.g., Winsauer, 1952).

Electrical conductivity tomography is a key technique in hydrogeophysics (Binley et al., 2015). Unfortunately, Eq. (1) remains the basis for the interpretation of resistivity data in a large number of publications in hydrogeophysics. Back in the $50 \mathrm{~s}$ and $60 \mathrm{~s}$ (e.g., Patnode and Wyllie, 1950, and Wyllie and Southwick, 1954), petroleum engineers recognized that a second contribution, called surface conductivity, was at play, especially in shaly formations. Waxman and Smits (1968) developed a simple equation written at this point as (see also Cremers and Laudelout, 1965, Rhoades et al., 1976, Mualem and Friedman 1991, Revil, 2017a, b),

$\sigma=\frac{1}{F} \sigma_{w}+\sigma_{S}$.

In Eq. (2), $\sigma_{S}\left(\mathrm{~S} \mathrm{~m}^{-1}\right)$ denotes this extra conductivity term called surface conductivity or surface conductance of the clay (e.g., Cremers et al., 1966). The form of Eq. (2) can be traced back to Patnode and Wyllie (1950) and Wyllie and Southwick (1954). In the seminal model of Waxman and Smits (1968), the surface conductivity is written as $\sigma_{S}=$ $\left(\hat{B} Q_{V}\right) / F$ where $\hat{B}$ (in the range 2.0 to $4.8 \times 10^{-8} \mathrm{~m}^{2} \mathrm{~V}^{-1} \mathrm{~s}^{-1}, \mathrm{Na}^{+}$, at $25^{\circ} \mathrm{C}$ ) denotes the apparent mobility for the charge carriers (called counterions) responsible for the surface conductivity (see also discussions in Revil et al., 1998). The term $Q_{V}$ (in $\mathrm{C} \mathrm{m}^{-3}$ ) denotes an excess of charge (of the counterions) per unit pore volume due to the cation exchange capacity of the surface of minerals. Clay minerals are characterized by

\footnotetext{
Intended for publication in Advances in Water Resources.

* Corresponding author at: Université Savoie Mont Blanc, ISTerre, Technolac, Bat. Belledonnes, rue du lac d'Annecy, 73370 Le Bourget-du-Lac, France.

E-mail addresses: andre.revil@univ-smb.fr (A. Revil), abdellahi.soueid-ahmed@univ-smb.fr (A. Soueid Ahmed), stephan.matthai@unimelb.edu.au (S. Matthai).
} 


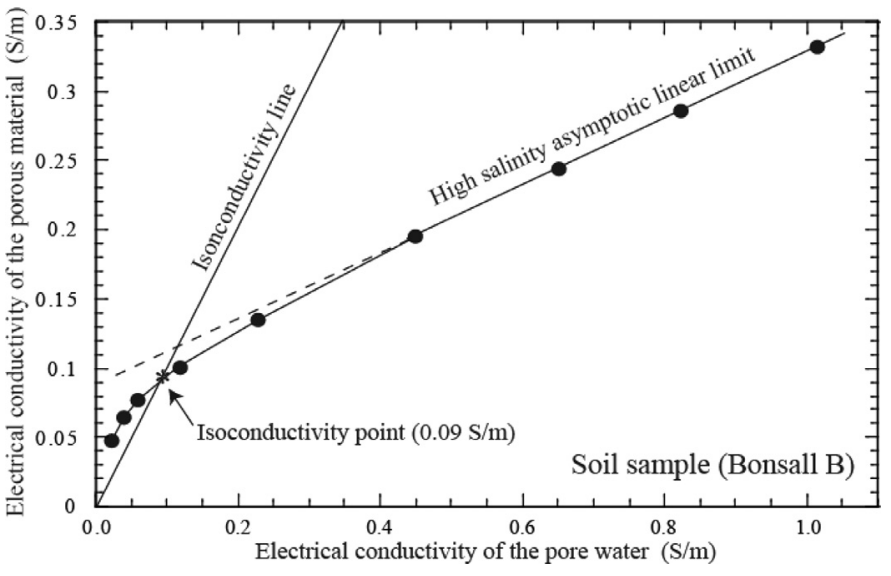

Fig. 1. Anatomy of the electrical conductivity curve of a porous body as a function of the pore water conductivity. This shows an example of non-linear behavior between the conductivity data of the porous material and the conductivity of the pore water. Data from Shainberg et al. (1980).

strong CEC values, but pure silica is characterized by a reactive mineral surface responsible for surface conductivity (see Revil et al., 2014). The surface conductivity is due to conduction in the electrical double layer (Stern and diffuse layers) coating the surface of these minerals.

Although Eq. (2) suggests a linear relationship between the conductivity of the porous body $\sigma$ and the pore water conductivity $\sigma_{w}$, Waxman and Smits (1968) recognized that, at low salinity, the actual behavior of $\sigma$ is non-linear. They observed that this non-linear behavior is especially pronounced for materials characterized by a high cation exchange capacity (CEC). To account for this observation, Waxman and Smits introduced a salinity dependent cation mobility $\hat{B}\left(\sigma_{w}\right)=$ $\hat{B}\left[1-0.6 \exp \left(-\sigma_{w} / 0.013\right)\right]$ (with $\sigma_{w}$ expressed in $\mathrm{S} \mathrm{m}^{-1}$ ). However, this explanation is unphysical since the mobility of the cations is not expected to decrease with decreasing salinity.

Many errors can be found in the literature regarding the improper use of electrical conductivity equations. Notably, when Eq. (1) is used while Eq. (2) should be applied instead, this results in unphysical values and dependencies of Archie's porosity exponent. For instance, Salem and Chilingarian (1999) observed (erroneously) that this exponent is salinity dependent although it is purely a textural parameter. The list of such errors is, unfortunately, very long both in petroleum engineering and in hydrogeophysics. That said, while Eq. (2) has increasingly been used in hydrogeophysics, we can wonder how well this equation models freshwater environments because it does not capture the low-salinity nonlinear behavior (e.g. Shainberg et al., 1980, for soils) - at least not in a physically meaningful way.

In the previous two papers of this series (Revil, 2017a, b), we develop a theory of ionic transport in porous media in unsaturated conditions. We kept the theory to the high salinity asymptotic behavior for which surface effects are treated as a perturbation of the transport in the connected pore space. In this situation, the transport of ions is mostly affected by the tortuosity of the bulk pore space. In the present paper, we show that the tortuosity affecting the transport of the ions changes with the salinity. The main goal of the present paper is to demonstrate to the hydrogeophysical community that beyond Eq. (2), there is another realm worth exploring for freshwater environments. It may be important for soils (Rhoades et al., 1976; Nadler, 1982; 1991; Binley et al., 2015), geosynthetic clay liners (Abuel-Naga and Bouazza, 2016), clay suspensions (van Olphen, 1957; van Olphen and Waxman, 1958), the geophysical monitoring of $\mathrm{CO}_{2}$ sequestration (Al Hagrey, 2012; Börner et al., 2013) and reactive transport modeling (Day-Lewis et al., 2017), and the study of gas hydrates and permafrost (Spangenberg, 2001; Priegnitz et al., 2015) to cite a few examples. We review knowledge about the non-linear behavior of electrical conductivity at low salinities (see for instance Bruggeman, 1935, Wyllie and Southwick, 1954, Bussian, 1983, Lima and Sharma, 1990, Schwartz et al., 1989a,b), starting with a volume averaging approach to a non-linear conductivity equation valid for any type of porous materials. Our analysis will start with the works of Johnson et al. (1986) and Johnson and Sen (1988). High and low salinity asymptotic limits can be rigorously derived and tied together using a Padé approximant (i.e., a ratio of polynomials). Then, we will explore how this equation can be used to explain the Wyllie and Southwick (1954), Waxman and Smits (1968), and dual water (Clavier et al., 1984) models. We will also compare the model to the differential effective medium solution for a pack of spheres coated by an electrical double layer and immersed in "background" (pore) water (see Bussian, 1983, Lima and Sharma, 1990). Finally, these models will be compared with numerical simulations at the pore scale at saturated and unsaturated conditions. We will also derive new expressions for the conductivity and new relationships between the models and check how these models compare with experimental data.

\section{Volume averaging approach}

Fig. 1 shows one example of a non-linear relationship between the total electrical and the pore water conductivity. We see that the conductivity curve is characterized by an isoconductivity point for which the conductivity of the porous medium is equal to that of the pore water, a property broadly analyzed in the colloidal science of clay suspensions (e.g., Street, 1963, Cremers and Laudelout, 1965, Shainberg and Levy, 1975) and porous media (Bussian, 1983; Lima and Sharma, 1990; Revil et al., 1998). In this section, we summarize the findings regarding the electrical conductivity of porous media and especially those from the original works of Johnson et al. (1986) and Johnson and Sen (1988) in an attempt to explain the non-linear behavior shown in Fig. 1. We start by considering two asymptotic limits for the conductivity equation corresponding at high salinities (i.e., higher than the salinity corresponding to the isoconductivity point) and at low salinities (i.e., for salinities much smaller than the salinity corresponding to the isoconductivity point).

\subsection{The formation factor}

We start our analysis by considering the local conductivity problem in the absence of an electrical double layer around the solid phase. The solid phase is insulating. The pore space is filled by an electrolyte of conductivity $\sigma_{w}$. The constitutive (local Ohm's law) and continuity equation for the current density are given by $\mathbf{j}=\sigma_{w} \mathbf{e}_{b}$ in $V_{p}$, (i.e., in the pore space) and $\nabla \cdot \mathbf{j}=0$ on $S$ (i.e., at the mineral water interface) where $\mathbf{e}_{b}=-\nabla \psi_{b}$ denotes the local electrical field $\left(\mathrm{V} \mathrm{m}^{-1}\right)$, and $\psi_{b}$ the local electrical potential (in $\mathrm{V}$ ), $\mathbf{j}$ denotes the local current density $\left(\mathrm{A} \mathrm{m}^{-2}\right.$ ). The subscript " $\mathrm{b}$ " refers to the fact that in this situation, the electrical field is governed by the distribution of the bulk conductances (Bernabé and Revil, 1995). In the absence of surface conductivity, the conduction problem reduces to:

$\nabla^{2} \psi_{b}=0$ in $V_{p}$

$\hat{\mathbf{n}} \cdot \mathbf{e}_{b}=0$ on $S$

$\psi_{b}=\left\{\begin{array}{l}\Delta \Psi \text { at } z=L \\ 0 \text { at } z=0\end{array}\right.$

where $V_{p}$ and $S$ denote the pore volume and (specific) interface area between the solid and the fluid respectively; $L$ (in $\mathrm{m}$ ) denotes the length of the cylindrical representative volume in the direction of the applied macroscopic electrical field $\mathbf{E}=-(\Delta \Psi / L) \tilde{\mathbf{z}}$ (in $\mathrm{V} \mathrm{m}^{-1}$ ), $\tilde{\mathbf{z}}$ denotes the unit vector in the direction of the electrical field, $\Delta \Psi$ corresponds to the difference of electrical potential between the end-faces of the representative volume, and $\hat{\mathbf{n}}$ denotes the unit vector normal to the surface of 
the grains. This boundary value problem can be written in terms of a normalized electrical potential $\Gamma_{b}$ for a cylindrical representative elementary volume of porous material (Pride, 1994), as follows:

$\nabla^{2} \Gamma_{b}=0$ in $V_{p}$

$\hat{\mathbf{n}} \cdot \nabla \Gamma_{b}=0$ on $S$

$\Gamma_{b} \equiv\left(\frac{\Delta \Psi}{L}\right)^{-1} \psi_{b}=\left\{\begin{array}{l}L \text { at } z=L \\ 0 \text { at } z=0\end{array}\right.$

In absence of surface conductivity, the formation factor $F=\sigma_{w} / \sigma$ (see Eq. (1)) is obtained by summing up the local Joule dissipation of energy (Johnson and Sen (1988); Revil and Cathles, 1999). The macroscopic dissipation of energy can be written as $D \equiv \mathrm{JE}$ while the local dissipation of energy is $d \equiv \mathbf{j e}_{b}$ where $\mathbf{J}$ and $\mathbf{j}$ denotes the macroscopic and local current densities, respectively. Therefore we have

$D=\frac{1}{V} \int_{V_{p}} d\left(\mathbf{e}_{b}\right) d V_{p}$

$\sigma\left(\frac{\Delta \Psi}{L}\right)^{2}=\frac{1}{V} \int_{V_{p}} \sigma_{w}\left|\mathbf{e}_{b}\right|^{2} d V_{p}$

$\frac{1}{F}=\left(\frac{\Delta \Psi}{L}\right)^{-2} \frac{1}{V} \int_{V_{p}}\left|\mathbf{e}_{b}\right|^{2} d V_{p}$,

$\frac{1}{F}=\frac{1}{V} \int_{V_{p}}\left|\nabla \Gamma_{b}\right|^{2} d V_{p}$

where $V$ is the total volume of the considered representative elementary volume and where $\sigma_{w}$ is considered constant over the pore space. This principle is always valid since the macroscopic dissipation of energy at the scale of a representative elementary volume is the sum of the local dissipations (here limited to the pore space). From Eq. (12), the inverse of the formation factor appears to correspond to an effective porosity (the connected porosity, a purely geometrical quantity, is $\phi=V_{p} / V$ ). For any type of porous material, this effective porosity is built by giving some weight to the throats and no weight to dead-ends of pores. This can be shown for instance for the material sketched in Fig. 2 for which the numerical simulation is performed at high salinity. The distribution of the $\Gamma_{b}$-field is shown in Fig. 3. This $\nabla \Gamma_{b}$-field is strong in pore throats and null in dead-ends (Section 6 below explains in detail how these computations are performed). This would remain true for any type of pore network, idealized or not. The formation factor goes to infinity as pores become disconnected (and very large near the percolation threshold). This paper does not focus on the specific physics of electrical conduction close to percolation.

\subsection{High salinity asymptotic limit}

Now we discuss the impact of an electrical double layer, coating the surface of the grains (Fig. 4a), contributing extra surface conductivity to the porous medium. We introduce $\Sigma_{S}$ (in S), the specific surface conductivity/conductance of the electrical double layer (in S) (see Fig. 4, and e.g., van Olphen, 1957, Johnson and Sen, 1988),

$\Sigma_{S}=\int_{0}^{\infty}\left(\sigma(x)-\sigma_{w}\right) d x$,

where $\sigma(x)$ denotes the local conductivity in the vicinity of the mineral surface / pore water interface (see details in Fig. 4b). Outside the electrical double layer, $\sigma(x)=\sigma_{w}$ where $x$ denotes the local coordinates normal to the pore water / solid interface. In the thin double layer assumption, the local electrical conductivity distribution is written as $\sigma(x)$ $=\sigma_{w}+\Sigma_{S} \delta(x)$ where $\delta(x)$ denotes the delta function characterizing the position of the mineral surface. To obtain the high salinity asymptotic behavior of the conductivity, we replace the conductivity of the pore a. Geometry
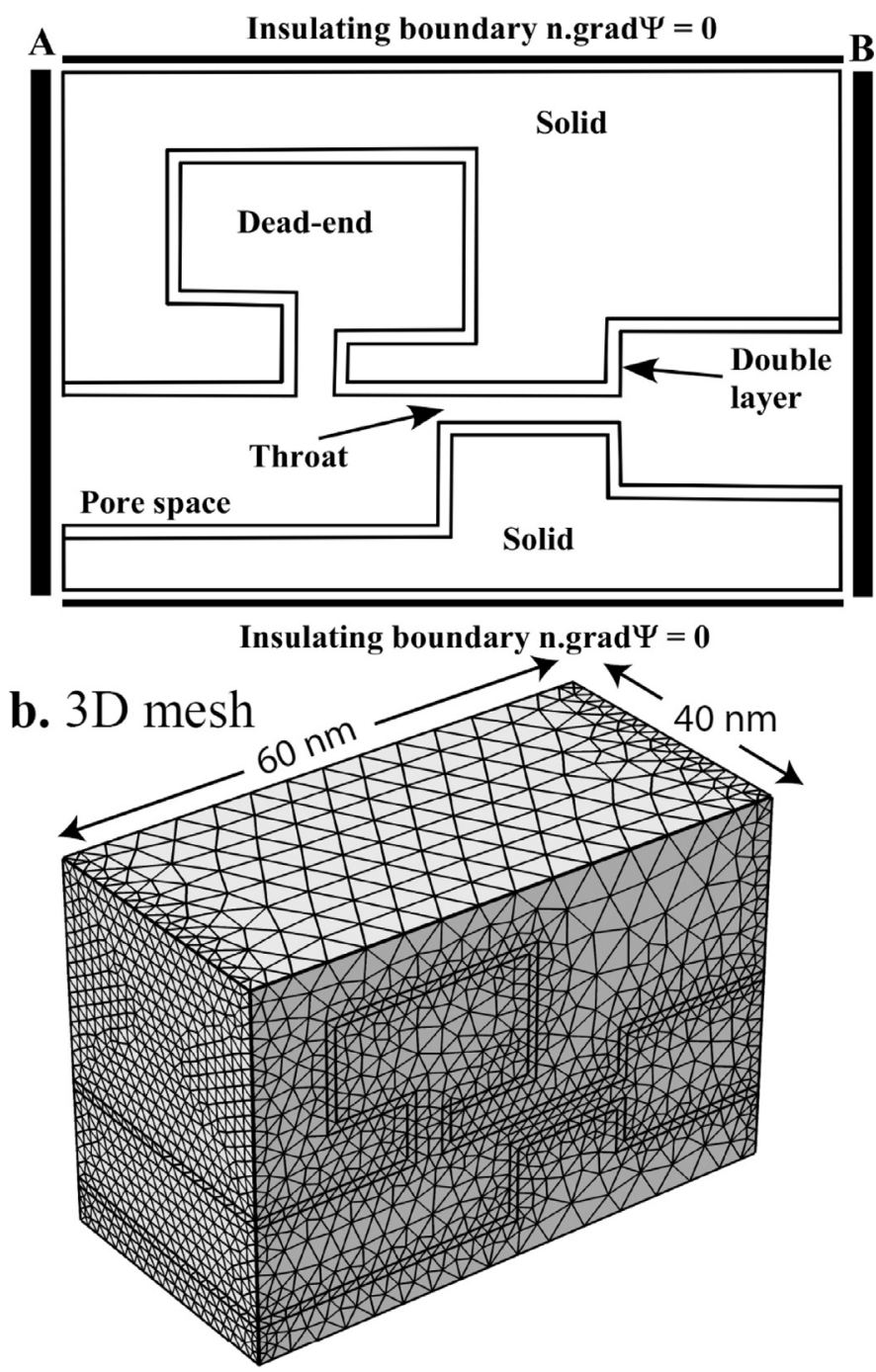

Fig. 2. Sketch of the first 3D porous material used to illustrate the conductivity problem. a. Geometry with boundary conditions. The material is made of an insulating mineral (null conductivity) coated by a conductive electrical double layer (conductivity of $1 \mathrm{~S} \mathrm{~m}^{-1}$ ). The (connected) pore space contains on deadend and one throat. The electrodes A and B are used to inject the current $I$ (in A) while all the other boundaries are insulating. The vector $\mathrm{n}$ denotes the normal unit vector to the external boundaries of the porous body and $\psi$ the electrical potential. b. Mesh used for the finite element calculations with Comsol Multiphysics of the Ohmic conductivity problem.

water $\sigma_{w}$ by the local conductivity $\sigma(x)$ assuming that in this high salinity limit, the electrical field remains roughly the same. This yields

$\sigma\left(\frac{\Delta \Psi}{L}\right)^{2}=\frac{1}{V} \int_{V_{p}} \sigma(x)\left|\mathbf{e}_{b}\right|^{2} d V_{p}$

At high salinity, the electrical field is controlled by the distribution of bulk conductances and we replace the local conductivity by its expression obtained with the thin double layer assumption, i.e., $\sigma(x)=$ $\sigma_{w}+\Sigma_{S} \delta(x)$. This yields (Johnson et al., 1986),

$\sigma\left(\frac{\Delta \Psi}{L}\right)^{2}=\frac{1}{V} \int_{V_{p}}\left[\sigma_{w}+\Sigma_{S} \delta(x)\right]\left|\mathbf{e}_{b}\right|^{2} d V_{p}$,
$\sigma\left(\frac{\Delta \Psi}{L}\right)^{2}=\frac{\sigma_{w}}{V} \int_{V_{p}}\left|\mathbf{e}_{b}\right|^{2} d V_{p}+\frac{\Sigma_{S}}{V} \int_{V_{p}} \delta(x)\left|\mathbf{e}_{b}\right|^{2} d V_{p}$, 


\section{$\Gamma_{b}$-distribution}

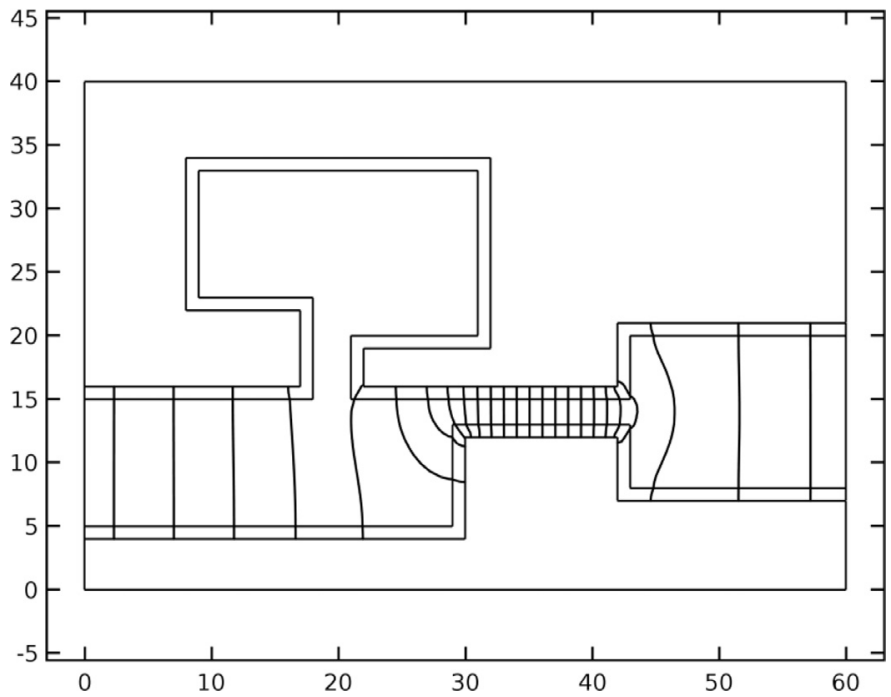

Fig. 3. Normalized potential $\Gamma_{b}$ (unitless) distribution obtained at high salinity $\left(\sigma_{w}=10 \mathrm{~S} \mathrm{~m}^{-1}\right)$. The local electrical field is very strong in the throat and equal to zero in the dead-end. The tortuosity of the conduction path is small. The dimensions are in $\mathrm{nm}$. For this material, we obtain $\Lambda=5.1 \mathrm{~nm}$ close to the size of the throat.

$\sigma\left(\frac{\Delta \Psi}{L}\right)^{2}=\frac{\sigma_{w}}{V} \int_{V_{p}}\left|\mathbf{e}_{b}\right|^{2} d V_{p}+\frac{\Sigma_{S}}{V} \int_{S}\left|\mathbf{e}_{b}\right|^{2} d S$,

$\sigma=\frac{\sigma_{w}}{F}\left(1+\frac{2}{\Lambda} \frac{\Sigma_{S}}{\sigma_{w}}+O\left(\frac{2}{\Lambda} \frac{\Sigma_{S}}{\sigma_{w}}\right)^{2}\right)$

using the Bachmann-Landau notation. From Eqs. (17) and (18), the length scale $\Lambda$ is defined by (Johnson et al., 1986),

$\frac{2}{\Lambda}=\frac{\int_{S}\left|\nabla \Gamma_{b}\right|^{2} d S}{\int_{V_{p}}\left|\nabla \Gamma_{b}\right|^{2} d V_{p}}$

The quantity $\Lambda$ appears fundamentally as an effective pore radius of the pore space, which can be eventually related to the permeability (Johnson et al., 1986; Bernabé and Revil, 1995).

\subsection{Low salinity asymptotic limit}

There is also a low salinity asymptotic limit of the conductivity problem for which the electrical field $\mathbf{e}$ is controlled by the distribution of the surface conductances. By low salinity, we mean salinities much smaller than that corresponding to the isoconductivity point. For such values, the electrical field takes the distribution $\mathbf{e}_{S}=-\nabla \psi_{S}\left(\psi_{S}\right.$ denotes the local electrical potential). Again the total Joule dissipation is the sum of the local contributions (Eq. (9)), i.e.,

$\sigma\left(\frac{\Delta \Psi}{L}\right)^{2}=\frac{\sigma_{w}}{V} \int_{V_{p}}\left|\mathbf{e}_{S}\right|^{2} d V_{p}+\frac{\Sigma_{S}}{V} \int_{S}\left|\mathbf{e}_{S}\right|^{2} d S$,

$\sigma=\frac{\sigma_{w}}{V} \int_{V_{p}}\left|\nabla \Gamma_{S}\right|^{2} d V_{p}+\frac{\Sigma_{S}}{V} \int_{S}\left|\nabla \Gamma_{S}\right|^{2} d S$,

$\sigma=\frac{\Sigma_{S}}{V} \int_{S}\left|\nabla \Gamma_{S}\right|^{2} d S\left[1+\frac{\sigma_{w}}{\Sigma_{S}} \frac{\int_{V_{p}}\left|\nabla \Gamma_{S}\right|^{2} d V_{p}}{\int_{S}\left|\nabla \Gamma_{S}\right|^{2} d S}\right]$,

and where the normalized low salinity potential $\Gamma_{S}$ is defined by,

$\Gamma_{S} \equiv\left(\frac{\Delta \Psi}{L}\right)^{-1} \psi_{S}$

\section{a. Sketch of the electrical double layer}

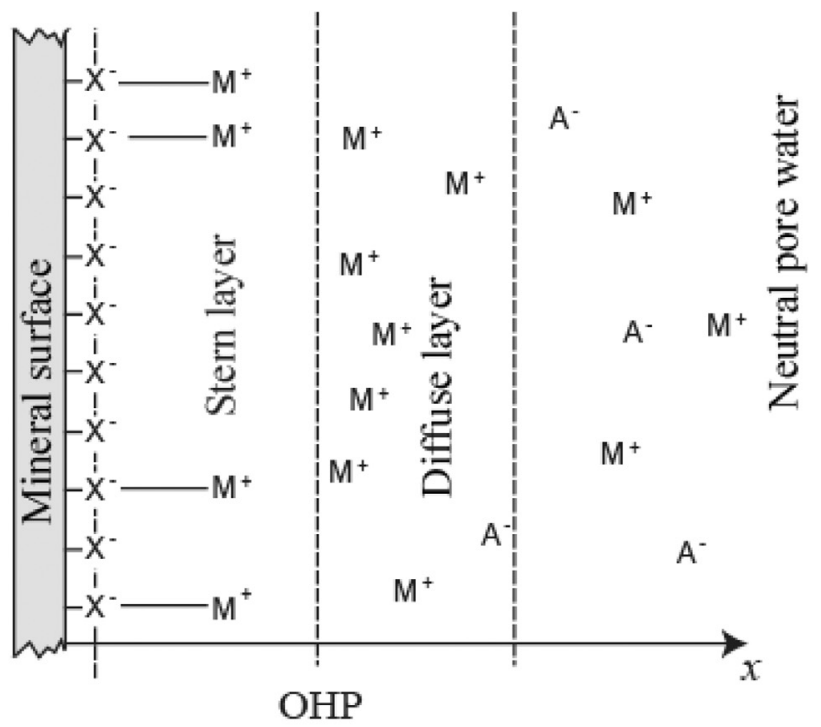

\section{b. Excess conductivity}

Local conductivity $\sigma(x)$

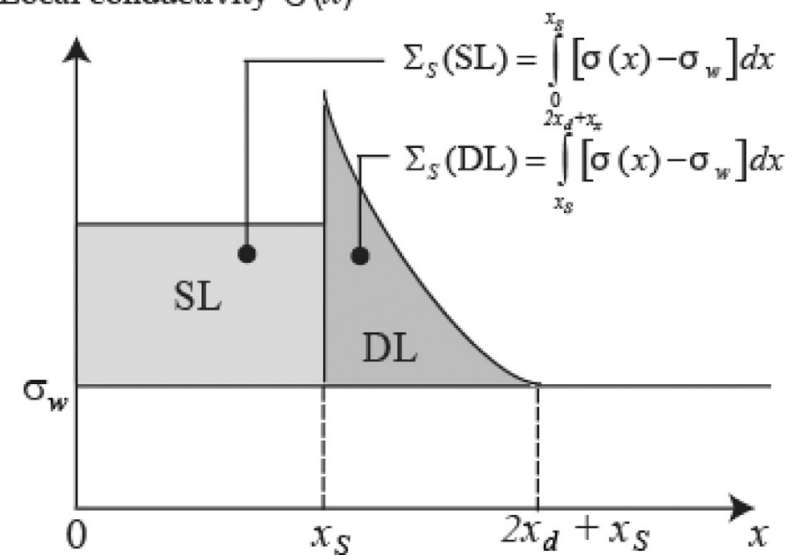

Fig. 4. Double layer and specific surface conductivity. a. Structure of the electrical double layer with the Stern and diffuse layers. OHP denotes the Outer Helmholtz Plane. b. Specific surface conductivity $\Sigma_{\mathrm{s}}$ (in S) (in grey) determined from the distribution of the local conductivity $\sigma(x)$. It comprises two contributions, one associated with the Stern layer (SL) of thickness $x_{s}$ and one with the diffuse layer (DL, thickness equal to twice the Debye screening length $x_{d}$, in $\mathrm{m}$ ).

From Eq. (22), the low-salinity asymptotic linear expression of the electrical conductivity becomes,

$\sigma=\frac{\Sigma_{S}}{f}\left(1+\frac{\lambda}{2} \frac{\sigma_{w}}{\Sigma_{S}}+O\left(\frac{\lambda}{2} \frac{\sigma_{w}}{\Sigma_{S}}\right)^{2}\right)$.

The two fundamental textural parameters appearing in Eq. (24) are given by,

$\frac{1}{f}=\frac{1}{V} \int_{S}\left|\nabla \Gamma_{S}\right|^{2} d S$

$\frac{2}{\lambda}=\frac{\int_{S}\left|\nabla \Gamma_{S}\right|^{2} d S}{\int_{V_{p}}\left|\nabla \Gamma_{S}\right|^{2} d V_{p}}$. 


\section{$\Gamma_{s}$-distribution}

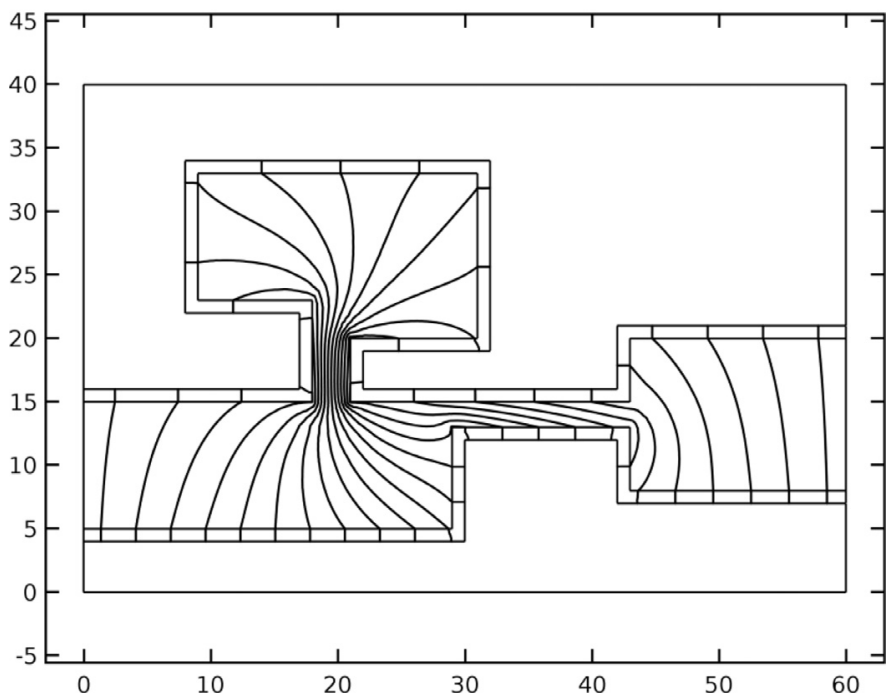

Fig. 5. Normalized potential $\Gamma_{S}$ (unitless) obtained at low salinity ( $\sigma_{w}=0.01 \mathrm{~S}$ $\mathrm{m}^{-1}$ ). Note that the electrical field is relatively uniform in the electrical double layer coating the surface of the solid phase because of its constant thickness. Since in this case the double layer has a uniform thickness and is continuous along the material, this prevents its polarization. The dimensions are given in $\mathrm{nm}$.

The new length scale $\lambda$ (in $\mathrm{m}$ ) appears to be a variant of the hydraulic radius $V_{p} / S$ weighted by the norm of the electrical field in absence of conductivity in the bulk pore space while the quantity $f$ (in $\mathrm{m}$ ) is a variant of the formation factor for surface conduction. For the material sketched in Fig. 2, the $\Gamma_{S}$-field is shown in Fig. 5 . We see that it appears rather uniform in the electrical double layer coating on the surface of the solid phase. Note that if the double layer is discontinuous, $f$ will go to infinity. In this case, the conductivity of the porous medium goes to zero when the salinity decreases to zero. We will come back on this point later in Sections 4 and 5, especially when analyzing another upscaling method called the differential effective medium theory.

An application of the Cauchy-Schwartz inequality applied to Eqs. (12) and (25) yields (Revil and Glover, 1997)

$F \geq \frac{1}{\phi}$

$f \geq \frac{V_{p}}{S} \frac{1}{\phi}$

where $\phi=V_{p} / V$ denotes the (connected) porosity. In connection with Archie's law $F=\phi^{-m}$, Eq. (27) implies that $m \geq 1$. We can postulate something equivalent to an Archie's law for the surface formation factor $f$, i.e., $f=\left(V_{p} / S \phi /\right)^{p}$ with $p \geq 1$. In addition, electrical conduction minimizes the Joule dissipation of energy (Revil and Glover, 1997) so

$\frac{1}{V} \int_{S} \Sigma_{S}\left|\mathbf{e}_{b}\right|^{2} d S \geq \frac{1}{V} \int_{S} \Sigma_{S}\left|\mathbf{e}_{S}\right|^{2} d S$,

$\frac{1}{V} \int_{V_{p}} \sigma_{w}\left|\mathbf{e}_{S}\right|^{2} d V_{p} \geq \frac{1}{V} \int_{V_{p}} \sigma_{w}\left|\mathbf{e}_{b}\right|^{2} d V_{p}$,

and in turn this yields the following inqualities (Revil and Glover, 1997)

$\frac{\lambda}{2 f} \geq \frac{1}{F}$

$\frac{2}{F \Lambda} \geq \frac{1}{f}$

$\lambda \geq \Lambda$
Eq. (31) appears as a consequence of Eq. (29) and (30). Eq. (29) implies that the slope of the $\sigma=g\left(\sigma_{w}, \Sigma_{S}\right)$ conductivity curve increases at low salinity and Eq. (30) implies that the $y$-intercept decreases with the salinity, therefore the curve $\sigma=g\left(\sigma_{w}, \Sigma_{S}\right)$ is concave. Another cause of convexity for the $\sigma=g\left(\sigma_{w}, \Sigma_{S}\right)$ curve (see Fig. 1) is coming from the dependence of $\Sigma_{S}$ with the salinity as discussed by Revil et al. (1998). Typically, we expect $\Sigma_{S}$ to increase due to sorption of the counterions in the electrical double layer (see Revil and Florsch, 2010).

\subsection{Surface and bulk tortuosities}

The bulk tortuosity of a porous material can be defined as (e.g., Pride, 1994),

$\alpha_{b}=F \phi=\frac{V_{p}}{\int_{V_{p}}\left|\nabla \Gamma_{b}\right|^{2} d V_{p}}$,

( $\geq 1$ ) and where the definition $\alpha_{b}=F \phi$ is from Wyllie and Rose (1950). A surface tortuosity can be also defined with respect to the current flow paths along the interface between the solid phase and the pore water phase as

$\alpha_{S}=\frac{S}{\int_{S}\left|\nabla \Gamma_{S}\right|^{2} d S}=f \phi \frac{S}{V_{p}}$.

$(\geq 1)$. These tortuosities are defined as dynamic quantities controlled by the distribution of the dynamic field $\Gamma$ (see Johnson et al., 1986, Pride, 1994). They are "dynamically" connected by the current lines (Johnson et al., 1986). In the definition of the tortuosities, the current lines are locally weighted by the norm of the electrical field and therefore tortuosities are not simple geometrical constructs. A new inequality between these two tortuosities is discussed in Appendix A.

\subsection{Conductivity expression based on a Padé approximant}

The previous high and low-salinity asymptotic limits can be used to forge a more general equation valid over the whole salinity range and solution of the Laplace problem. For a Padé approximant (a ratio of polynomials), we need to find the coefficients of the function $g(x)$ (with $x=\Sigma_{S} / \sigma_{w}$, Dukhin number), called a Padé approximant, such as,

$\sigma=\sigma_{w} g(x)$,

$g(x)=\frac{b+c x+d x^{2}}{1+a x}$,

and satisfying,

$\sigma=\sigma_{w}\left[\frac{1}{F}+\frac{2}{\Lambda F} x+O\left(\frac{2}{\Lambda} x\right)^{2}\right]$,

$\sigma=\sigma_{w}\left[\frac{\lambda}{2 f}+\frac{1}{f} x+O\left(\frac{\lambda}{2} \frac{1}{x}\right)^{2}\right]$,

at high $\left(2 x / \Lambda=2 \Sigma_{S} / \sigma_{w} \Lambda \ll 1\right)$ and low $\left(2 x / \lambda=2 \Sigma_{S} / \sigma_{w} \lambda \gg 1\right)$ salinities, respectively. We have $F=[g(0)]^{-1}$. Eq. (37) is chosen as being the simplest Padé approximant (i.e., with the lowest order polynomials) leading to the asymptotic linear relationships (38) and (39). Indeed, expanding Eq. (37) to its asymptotic limits yields the following results (Appendix B)

$a=\frac{\frac{2}{\Lambda}}{\frac{\lambda F}{2 f}-1}$,

$c=\frac{\frac{2}{\Lambda}}{F-\frac{2 f}{\lambda}}$,

$b=1 / F$, 
$d=a / f$.

When interpreting conductivity data collected at different salinities, we fit the coefficient $a, b, c$, and $d$. Then we need to invert Eqs. (40)-(43) to get the fundamental textural parameters of interest. This yields

$\Lambda=2 /(c / b-a)$,

$\lambda=2 c / d$,

$F=1 / b$,

$f=a / d$.

Note also that the isoconductivity point corresponds to the conditions $\sigma=\sigma_{w}$, thus to $g(x)=1$. This yields in turn the following secondorder equation $d x^{2}+(c-a) x+b-1=0$ and the positive root of this equation can be easily determined to fulfill the condition.

A very clear physical meaning of Eq. (37), i.e., the general conductivity equation forged with the Padé approximant, can be given now as follow. First Eq. (37) is rewritten as,

$g(x)=\frac{x}{A x+B}+C x+D$,

where the four textural parameters are defined as

$A=\frac{1}{\frac{\lambda}{2 f}-\frac{1}{F}}$,

$B=\frac{1}{\frac{2}{\Lambda F}-\frac{1}{f}}$,

$C=\frac{1}{f}$

$D=\frac{1}{F}$.

Under this form, Eqs. (48)-(52) exhibit some form of symmetry. Using Eq. (36) together with $x=\Sigma_{S} / \sigma_{w}$, we can write the conductivity of the porous material as

$\sigma=\frac{\Sigma_{S} \sigma_{w}}{A \Sigma_{S}+B \sigma_{w}}+C \Sigma_{S}+D \sigma_{w}$.

Introducing the conductivity of the grains as

$\sigma_{s s}=\frac{1}{m} \frac{2}{\Lambda F} \Sigma_{S}$,

(a rigorous demonstration for this equation will be given below in Section 4) into Eq. (53) yields,

$\sigma=\frac{\sigma_{s s} \sigma_{w}}{A \sigma_{S}+B^{\prime} \sigma_{w}}+C^{\prime} \sigma_{s s}+D \sigma_{w}$

with $B^{\prime}=2 B /(m \Lambda F)$ and $C^{\prime}=C(m \Lambda F / 2)$. In this form, the conductivity model corresponding to Eq. (55) has exactly the same structure than the model developed by Wyllie and Southwick (1954) (see also Sauer et al., 1955) for which $A, B^{\prime}, C^{\prime}$ and $D$ were just unspecified textural properties (see also Lima et al., 2010, their Eq. 1). The model of Wyllie and Southwick (1954) assumes three electrical resistances working in parallel. They are (1) a resistance consisting of grains in electrical contact with each other; (2) a resistance consisting of the pore water located in the connected pore space; and (3) a resistance consisting of grains and pore water in series with each other (a complete discussion can also be found in Bussian, 1983). Our approach represents the first unification of the empirical model of Wyllie and Southwick with the model derived with the volume-averaging technique.

\section{Connection with other empirical models}

Here our goal is to show the connections between the previous model and empirical models such as the dual water model and the Waxman and Smits model. The dual water concept was originally proposed by Clavier et al. (1984) to emphasize the volume occupied by the electrical double layer in the expression of the surface conductivity of a porous material. In other words, the bulk conductivity and the surface conductivity should be associated with the volume fraction of porosity to which they correspond (double layer water and far water in the terminology of Clavier et al., 1984, also abusively called bound water and free water in a number of papers). The dual water concept introduced in this paper is coming directly from the expression of the specific surface conductivity, see Eq. (13). Integrating Eq. (13) over the thickness of the electrical double layer, we can break the specific surface conductivity into two terms,

$\Sigma_{S}=Q_{S} B-\sigma_{w} x_{s}$,

where $Q_{S}$ and $x_{S}$ denote the surface charge density of the electrical double layer (Stern and diffuse layers) and its thickness, respectively. According to et al. (2017a, b, c), the effective mobility $B$ can be written as,

$B=\beta_{(+)}^{S} f_{Q}+\beta_{(+)}\left(1-f_{Q}\right)$,

where $\beta_{(+)}^{S}$ and $\beta_{(+)}$denote the mobility of the counterions in the Stern and diffuse layers, respectively (we expect $\beta_{(+)}^{S}<\beta_{(+)}$since the Stern layer is much more packed than the diffuse layer and the degrees of freedom are smaller). Therefore $B$ is a composite of the mobility of the counterions in the Stern and diffuse layers weighted by the relative fractions of the counterions in these two layers (Revil et al., 2017c).The quantities $f_{Q}$ and $\left(1-f_{Q}\right)$ represent the fractions of charge carriers in the Stern later and diffuse layer, respectively. The quantity $f_{Q}$ is called the partition coefficient. Typically we have $B=1.49 \times 10^{-8} \mathrm{~m}^{2} \mathrm{~s}^{-1} \mathrm{~V}^{-1}$. With a charge density $Q_{S}=5$ charges $\mathrm{nm}^{-2}\left(0.81 \mathrm{C} \mathrm{m}^{-2}\right)$, this yields a value of $\Sigma_{S} \approx Q_{S} B=1.2 \times 10^{-8} \mathrm{~S}$. A charge density $Q_{S}=2$ charges $\mathrm{nm}^{-2}$ $\left(0.32 \mathrm{C} \mathrm{m}^{-2}\right)$ yields a value of $\Sigma_{S} \approx Q_{S} B=5 \times 10^{-9} \mathrm{~S}$.

At high salinity, the thickness of the double layer would reduce to the thickness of the Stern layer (Clavier et al., 1984). The quantities

$Q_{S} f_{Q}=e \Gamma_{(+)}^{S}$,

$Q_{S}\left(1-f_{Q}\right)=e \Gamma_{(+)}^{d}$,

denote the charge density in the Stern and diffuse layers, respectively and $\Gamma_{(+)}^{S}$ and $\Gamma_{(+)}^{d}$ denote the surface charge density of the counterions in the Stern and diffuse layers, respectively. A complete electrochemical analysis of this problem is out of the scope of the present paper.

Now we can introduce Eq. (56) into the high salinity asymptotic equation (see Eq. (18)),

$\sigma=\frac{1}{F}\left[\sigma_{w}+\frac{2 x_{s}}{\Lambda}\left(\frac{Q_{S} B}{x_{s}}-\sigma_{w}\right)+\ldots\right]$,

$\sigma=\frac{1}{F}\left[\sigma_{w}\left(1-\frac{2 x_{s}}{\Lambda}\right)+\frac{2}{\Lambda} Q_{S} B+\ldots\right]$,

$\sigma=\frac{\left(1-\frac{2 x_{s}}{\Lambda}\right)}{F}\left[\sigma_{w}+\frac{2 x_{s}}{\Lambda} \frac{Q_{S} B}{\left(1-\frac{2 x_{s}}{\Lambda}\right)}+\ldots\right]$,

and we can introduce apparent textural quantities as

$\sigma=\frac{1}{F_{a}}\left[\sigma_{w}+\frac{2}{\Lambda_{a}} Q_{S} B+\ldots\right]$,

where an apparent formation factor and an apparent pore size can be defined as

$F_{a}=\frac{F}{\left(1-\frac{2 x_{s}}{\Lambda}\right)}$, 
$\Lambda_{a}=\Lambda\left(1-\frac{2 x_{s}}{\Lambda}\right)=\Lambda-2 x_{s}$

We observe therefore the appearance of a dual water correction term $\left(1-2 x_{s} / \Lambda\right)$ in the definition of the apparent formation factor and apparent effective pore size. This correction term is however slightly distinct from the dual water correction term developed by Clavier et al. (1984), which is based on the relative volume of the double layer with respect to the entire pore space volume. We will come back on this point later. In our case, what matters is the relative size of the double layer with respect to the effective pore size (which is actually a measure of the size of the throats of the porous material, see Johnson et al., 1986; Bernabé and Revil, 1995). The quanties $F_{a}$ and $\Lambda_{a}$ correspond to the formation factor and apparent effective pore size obtained if the double layer would be excluded from the pore space. They are also the quantities that can be taken from the high salinity asymptotic curve at high salinities.

To demonstrate the connection between the previous model and the dual water and the Waxman and Smits models, we first start with a relationship between the length scale $\Lambda$ and the surface area per pore volume. Following Johnson et al. (1986), we have,

$\frac{2}{\Lambda}=-\frac{d \ln F}{d \ln \phi} \frac{S}{V_{p}}$

We can empirically relate $F$ to $\phi$ using Archie's law (see Archie, 1942), i.e., $F=\phi^{-m}$ where $m$ denotes the porosity exponent as mentioned above. Therefore Eq. (66) can be written as,

$\frac{2}{\Lambda} \approx m \frac{S}{V_{p}}$.

Starting with the high salinity approximation, Eq. (18), and using Eq. (67) we have

$\sigma=\frac{1}{F}\left[\sigma_{w}+m \frac{S}{V_{p}}\left(Q_{S} B-\sigma_{w} x_{s}\right)\right]$.

The next approximation is in the relationship between the specific surface conductivity and the excess of charge $Q_{V}$ or the cation exchange capacity (CEC, expressed in $\mathrm{C} \mathrm{kg}^{-1}$ or sometimes in mMol equivalent charge per gram of solid phase). The relationship between the charge per unit volume and the charge per unit surface area is given by $Q_{V}=Q_{S} S / V_{p}$ for the total volumetric charge density. The surface charge density $Q_{S}$ is roughly equivalent to 3 elementary charge per $\mathrm{nm}^{2}\left(\sim 0.48 \mathrm{C} \mathrm{m}^{-2}\right.$, see Revil et al. 2017a, b). The total volumetric charge density $Q_{V}$ is written as a function of the cation exchange capacity as (e.g., Waxman and Smits, 1968),

$Q_{V}=\rho_{g}\left(\frac{1-\phi}{\phi}\right) \mathrm{CEC}$,

and it follows,

$\sigma=\frac{1}{F}\left[\sigma_{w}\left(1-m \frac{S}{V_{p}} x_{s}\right)+m Q_{V} B\right]$,

$\sigma=\frac{\left(1-m \frac{S}{V_{p}} x_{s}\right)}{F}\left[\sigma_{w}+\frac{m Q_{V} B}{\left(1-m \frac{S}{V_{p}} x_{s}\right)}\right]$.

Following Clavier et al. (1984), we introduce the following quantity $v_{Q}$

$v_{Q}=\frac{m x_{s}}{Q_{S}}$,

$v_{Q} Q_{V}=m x_{s} \frac{S}{V_{p}}$.

In the dual water layer model, the quantity $v_{Q} Q_{V}$ denotes the volume fraction of the pore space occupied by the electrical double layer. Using
Eqs. (71)-(73), we obtain the dual water equation derived empirically by Clavier et al. (1968)

$\sigma=\frac{\left(1-v_{Q} Q_{V}\right)}{F}\left[\sigma_{w}+\frac{Q_{V} \hat{B}}{\left(1-v_{Q} Q_{V}\right)}\right]$.

with $\hat{B}=m B$. In the paper of Clavier et al. (1968), the quantity (1$\left.v_{Q} Q_{V}\right)$ is replaced by $\left(1-\alpha v_{Q} Q_{V}\right)$ with $\alpha$ denotes the ratio between the double layer thickness (diffuse plus Stern layers) to the thickness of the Stern layer. We have $\alpha=1$ at high salinities and $\alpha>1$ at low salinities. This salinity effect can explain some of the curvature observed at low salinities. In absence of dual water correction, this equation resumes to the high salinity asymptotic limit of the Waxman and Smits (1968) equation:

$\sigma=\frac{1}{F}\left[\sigma_{w}+m B Q_{V}\right]$,

where $m$ can be eventually encapsulated into an apparent mobility $\hat{B}=$ $m B$. Typically we have $B=1.49 \times 10^{-8} \mathrm{~m}^{2} \mathrm{~s}^{-1} \mathrm{~V}^{-1}$ and then, taking $m$ $=2$ yields $\hat{B} \approx 3 \times 10^{-8} \mathrm{~m}^{2} \mathrm{~s}^{-1} \mathrm{~V}^{-1}$ consistent with the values given in Waxman and Smits (1968).

\section{Use of the differential effective medium theory}

We consider now a particular class of porous media made of insulating grains entirely immersed in the background pore water. This means that the grains do not touch anymore and that the double layers are not interconnected. The solution of the differential effective medium scheme yields the following expression for the electrical conductivity of the material (Bruggeman, 1935; Hanai. 1960a,b, Sen et al., 1981 and Appendix C),

$\sigma=\sigma_{w} \phi^{m}\left(\frac{1-\sigma_{s s} / \sigma_{w}}{1-\sigma_{s s} / \sigma}\right)^{m}$.

which reduces to Eq. (1) with $F=\phi^{-m}$ in absence of grain conductivity, i.e., for $\sigma_{s s}=0$ (Bussian, 1983; Lima and Sharma, 1990). In saturated conditions and above the so-called isoconductivity point characterized (i.e., for $\sigma_{w} \geq \sigma_{s s}$ ), Eq. (76) has the following closed-form solution (Revil et al., 1998; Revil 2000),

$\sigma \approx \frac{\sigma_{w}}{F}\left[F \mathrm{X}+\frac{1}{2}(1-\mathrm{X})\left(1-\mathrm{X}+\sqrt{(1-\mathrm{X})^{2}+4 F \mathrm{X}}\right)\right]$,

where $F=\phi^{-m}$ denotes the formation factor and the Dukhin number (dimensionless) is defined by Revil et al. (1998),

$\mathrm{X} \equiv \frac{\sigma_{s s}}{\sigma_{w}}$.

A high salinity asymptotic limit can be obtained directly from Eq. (76) using Newton binomial expansion (Bussian, 1983)

$\sigma=\frac{1}{F} \sigma_{w}\left[1+m(F-1) X+O(X)^{2}\right]$

or alternatively,

$\sigma \approx \frac{\sigma_{w}}{F}+m\left(1 \frac{1}{F}\right) \sigma_{s s}$

Note that usually (except for colloidal suspensions) $F \gg 1$ (i.e., $(1-1 / F) \approx 1)$. From Eq. (80), it is also clear that the surface conductivity in Eq. (2) is given by $\sigma_{S} \approx m \sigma_{s s}$. A comparison with the high salinity asymptotic limit obtained using the volume averaging approach reveals a simple relationship between the two scaling factors $x$ and $X$,

$m X=\frac{2}{\Lambda F} x$.

From this relationship, we can connect the conductivity of the grains and the specific surface conductivity $\Sigma_{S}$ (used in Section 2.5 above)

$\sigma_{s s}=\frac{1}{m} \frac{2}{\Lambda F} \Sigma_{S}$. 
Using the expression of $\Lambda$ as a function of the surface per pore volume ratio and the expression of the specific surface conductance $\Sigma_{S}$, we have

$\sigma_{s s}=\frac{1}{F} \frac{S}{V_{p}}\left(Q_{S} B-\sigma_{w} x_{s}\right)$,

$\sigma_{s s}=\frac{1}{F}\left(Q_{V} B-\frac{S}{V_{p}} x_{s} \sigma_{w}\right)$.

This completes the work of Bussian (1983) by providing an explicit relationship between the conductivity of the solid phase and the cation exchange capacity, which was not achieved in his paper. Neglecting the correction term (i.e., the second term in the parenthesis of Eq. (84)), the conductivity of the grains is given by

$\sigma_{s s} \approx \frac{1}{F \phi}(1-\phi) B \rho_{g}$ CEC.

If the grains are round, we have $F \phi \approx 3 / 2$ (i.e., the tortuosity required for the flow lines to go around a sphere) and for small porosities (say smaller than $30 \%$ ) we have therefore

$\sigma_{s s} \approx \frac{2}{3} B \rho_{g} \mathrm{CEC}$.

This equation makes a lot of sense since it is equivalent of having the charge of the double layer divided by the volume of grain times the mobility of the counterions.

Note that for $\mathrm{X}=1$ (isoconductivity point), Eqs. (76) or (77) yields,

$\sigma(\mathrm{X}=1)=\sigma_{w}=\sigma_{s s}$.

In other words, the isoconductivity point is characterized by an equality between the conductivity of the medium filled with pore water and the conductivity of the solid phase coated by the electrical double layer. From Eq. (81), the isoconductivity point can also be written as a condition between the pore water conductivity and the specific surface conductivity and corresponds therefore to the condition $\sigma_{w}=$ $2 \Sigma_{S} /(m \Lambda F)$. Below the isoconductivity point (i.e., $\left.\sigma_{w} \leq \sigma_{s s}\right)$, Eq. (76) can be also be written as (Appendix C),

$\sigma=\sigma_{w} \phi^{\frac{m}{1-m}}\left(\frac{1-\sigma_{w} / \sigma_{s s}}{1-\sigma / \sigma_{s s}}\right)^{\frac{m}{1-m}}$.

The low-salinity equation for the electrical conductivity is (Revil, 2000),

$\sigma \approx \frac{\sigma_{w}}{g}\left[g \mathrm{X}+\frac{1}{2}(1-\mathrm{X})\left(1-\mathrm{X}+\sqrt{(1-\mathrm{X})^{2}+4 g \mathrm{X}}\right)\right]$,

with a low salinity formation factor $g=\phi^{m /(1-m)}=F^{1 /(m-1)} \geq 1$ (with $m=2, g=F)$. When surface conductivity dominates, we obtain

$\lim _{\mathrm{X} \gg 1} \sigma=g \sigma_{w}\left[1+O\left(\mathrm{X}^{-1}\right)\right]$,

$\lim _{\mathrm{X} \gg 1}\left(\frac{1}{\sigma}\right)=\frac{1}{g \sigma_{w}}\left[1+\frac{m}{m-1}(g-1) \frac{1}{\mathrm{X}}+O\left(\mathrm{X}^{-2}\right)\right]$,

where Eq. (91) can also be obtained directly from Eq. (88) using Newton binomial expansion (Lima and Sharma, 1990). From Eq. (90), we see that the conductivity of the porous material decreases linearly with the conductivity of the pore water at very low salinities down to zero (very high Dukhin number X). Indeed in the differential effective medium theory, the grains are not touching each other so if the conductivity of the water phase is going to zero, the conductivity of the material is also going to zero. We connect now the low salinity asymptotic limit with the one obtained by the volume averaging approach, see Eq. (2). This yields,

$(f, \lambda) \rightarrow+\infty$

$\frac{\lambda}{2 f}=g=\phi^{\frac{m}{1-m}}$.
The condition $f \rightarrow+\infty$ is directly obtained by combining Eqs. (91) and (25) while the condition $\lambda \rightarrow+\infty$ is obtained from the inequality $\lambda \geq 2 f / F$ with $F$ finite. The result $(f, \lambda) \rightarrow+\infty$ is not surprising and appears as a direct consequence of the fact that the grains (with their double layer) are not touching each other in this model. At low salinities, the conductivity $\sigma_{s s}=\Sigma_{S} /(m f)$ should therefore go to zero. In other words the grains appear as insulators. In a real porous medium, however, the diffuse layers overlap and the grains are therefore in electrical contact with one another, implying that the differential effective medium theory is not correct at very low salinities since in a real porous material.

\section{Effect of saturation}

We write $s_{\mathrm{w}}$ the saturation of a second immiscible fluid phase acting as an insulator (e.g. air or a non-wetting oil). The conductivity equations should be consistent with Archie's second law and the fact that the charge per unit volume should scale as

$Q_{V} \rightarrow Q_{V} / s_{w}$

(e.g., Waxman and Smits, 1968). This yields the following change of variables,

$\frac{1}{F} \rightarrow \frac{1}{F} s_{w}^{n}$

$\Lambda \rightarrow \Lambda s_{w}$.

At the opposite, the surface formation factor $f$ should be independent of the saturation since it does not involve any integrals over the pore volume. A similar analysis can be done for the differential effective medium approach. This yields,

$$
\begin{aligned}
\sigma= & \frac{\sigma_{w}}{F} s_{w}{ }^{n}\left[F s_{w}{ }^{-n} \mathrm{X}\left(s_{w}\right)+\frac{1}{2}\left(1-\mathrm{X}\left(s_{w}\right)\right)\right. \\
& \left.\left(1-\mathrm{X}\left(s_{w}\right)+\sqrt{\left(1-\mathrm{X}\left(s_{w}\right)\right)^{2}+4 F s_{w}{ }^{-n} \mathrm{X}\left(s_{w}\right)}\right)\right]
\end{aligned}
$$

where the Dukhin number is given by

$\mathrm{X}\left(s_{w}\right)=\frac{1}{m} \frac{2}{\wedge\left(s_{w}\right) F\left(s_{w}\right)}\left(\frac{\sum_{S}}{\sigma_{w}}\right)$,

$\mathrm{X}\left(s_{w}\right)=s_{w}^{n-1} \mathrm{X}$.

This formulation is consistent with the work done by Greve et al. (2013) who analyzed a large database of experimental data at partial saturations to conclude that the Dukhin number should scaled according to Eq. (99).

\section{Comparison with experiments}

\subsection{Numerical experiments}

We follow here some recent pore level modeling approaches to determine the electrical conductivity of porous media (e.g., Torskaya et al., 2014; Shabro et al., 2014). In the present case, we use a synthetic porous material to illustrate the conductivity behavior in saturated and unsaturated conditions. The computations are made with Comsol Multiphysics using the finite element method. The simulations are aimed to represent a pure Ohmic conductivity experiment with no polarization of the material. We solve the local problem corresponding to $\nabla \cdot[\sigma(x) \nabla \psi]=$ 0 with the boundary conditions given by Eqs. (4) and (5) and where $\sigma(x)$ is the local electrical conductivity. The local electrical conductivity is piecewise constant. Indeed, it is constant in the bulk pore space $\left(\sigma(x)=\sigma_{w}\right.$, conductivity of the pore water) outside the electrical double layer and it is constant in the layer coating the surface of the grains and representing the electrical double layer $\sigma(x)=\sigma_{s}$. We use insulating boundary conditions on the external surfaces except for the position of 


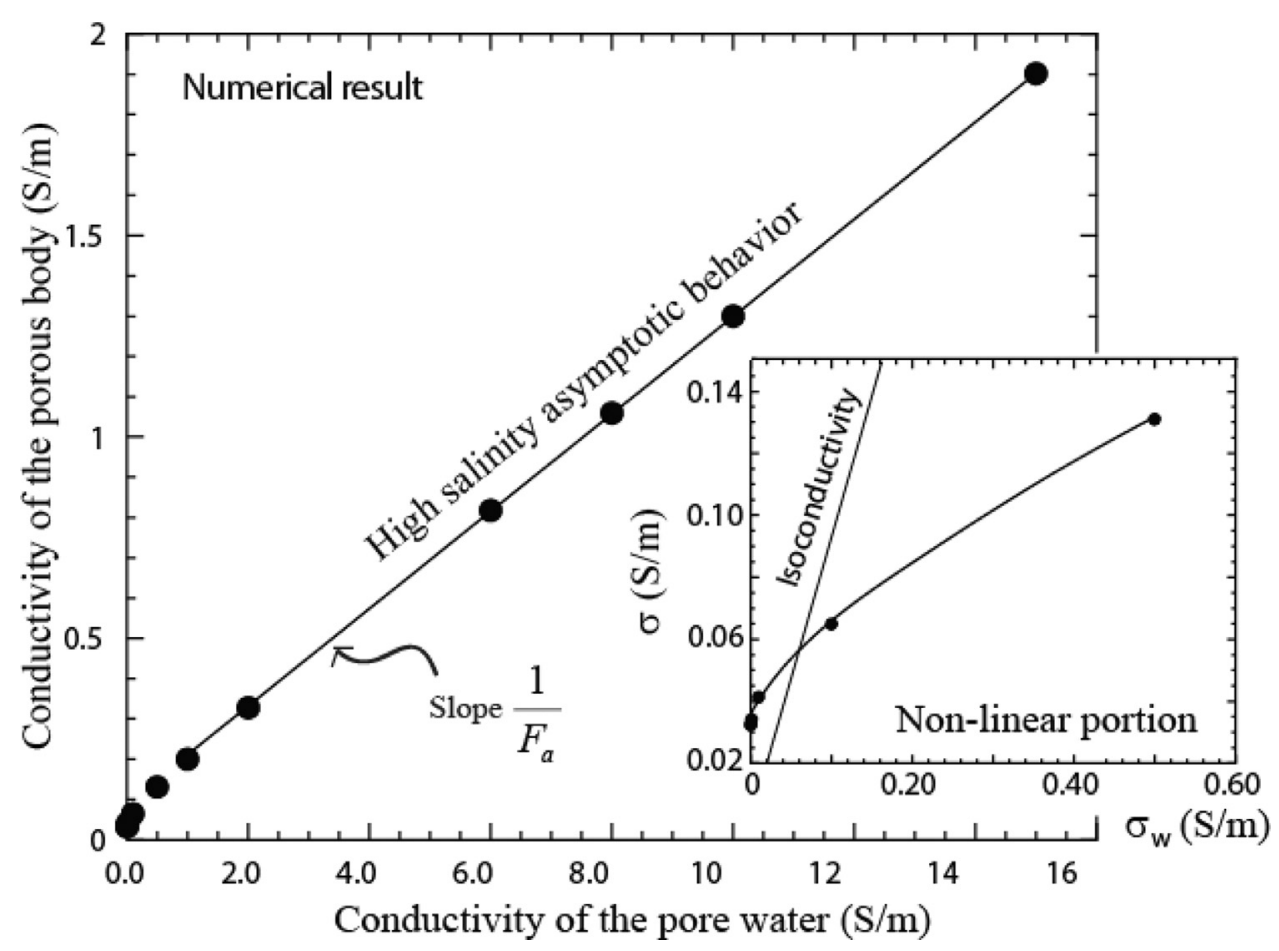

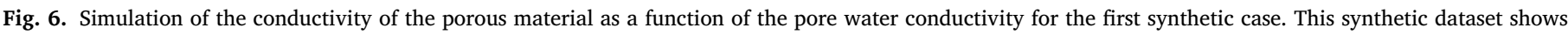

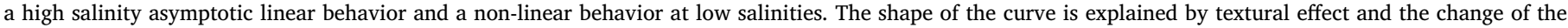
tortuosity with the distribution of the conductive paths when the salinity decreases. The isoconductivity point is characterized by the condition $\sigma_{w}=2 \Sigma_{S} /(m \Lambda F)$.

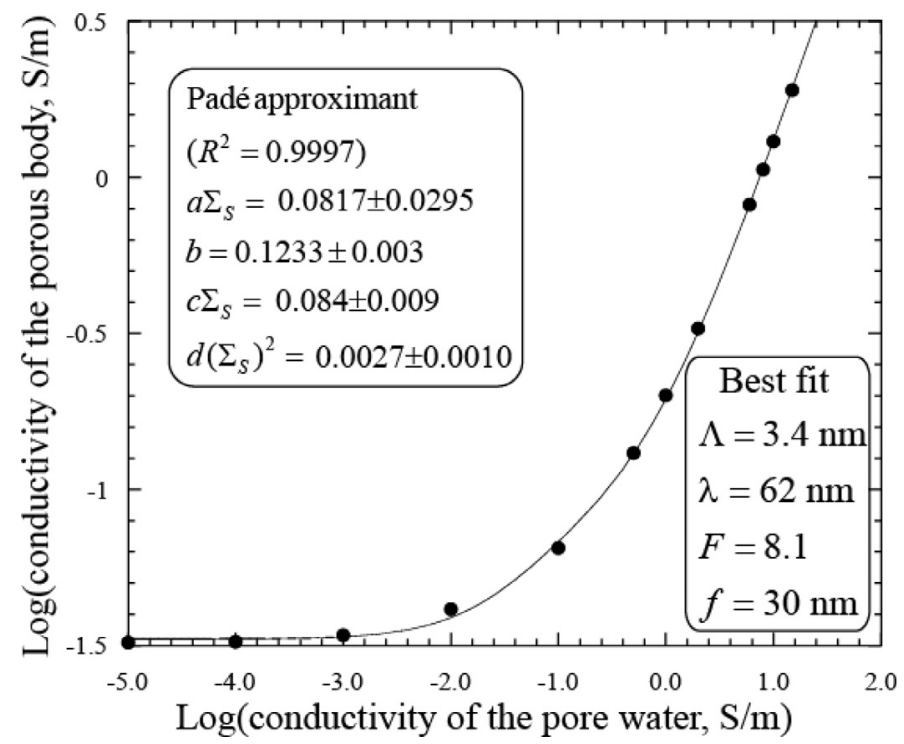

Fig. 7. Best fit in a log space of the Padé approximant (non-linear model) used to represent the conductivity of the porous material shown in Fig. 2 as a function of the pore water conductivity for a restricted range of salinity. Note that a fit done in a linear-linear space between the conductivity of the material versus the conductivity of the pore water does not provide correct estimates of the model parameters. With the exception of $\lambda$, the other three textural parameters are rather well-estimated. We check that $\lambda \geq \Lambda$ and $\lambda \geq 2 f / F$.

the electrodes A and B used to inject and retrieve the electrical current (Fig. 2).

The investigated porous medium corresponds to a micro-porous material such as clay-rich soils with a throat, a dead-end, and the surface of the grains coated by an electrical double layer (Fig. 2a). The length of this porous body is $60 \mu \mathrm{m}$, its height $40 \mathrm{~nm}$, and its thickness $30 \mathrm{~nm}$. The mesh contains 85,016 quadratic elements (Fig. 2b). Its porosity $\phi$

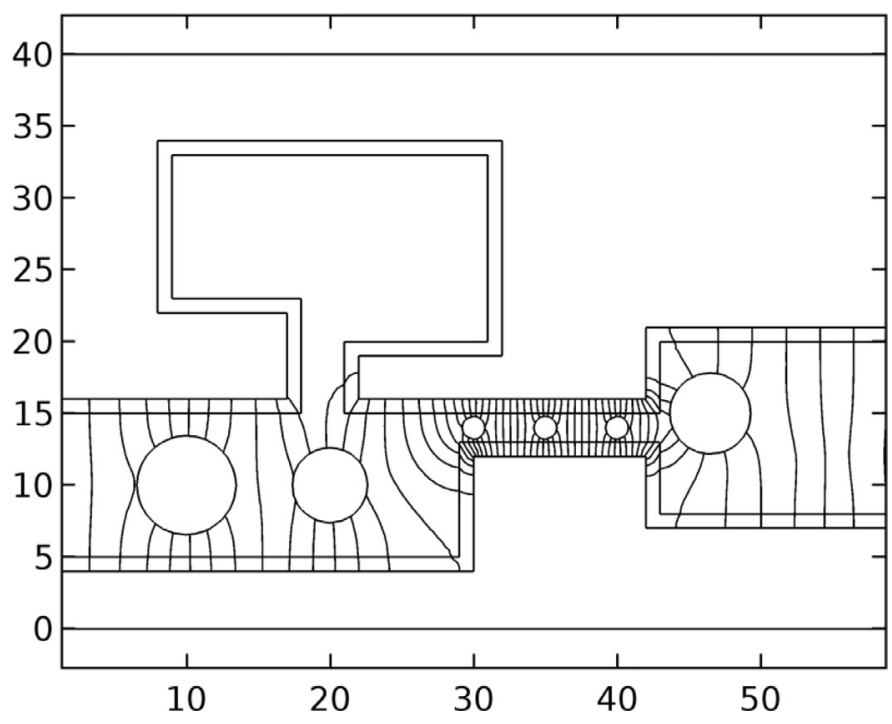

Fig. 8. Cross-section of the porous material shown in Fig. 2 showing the distribution of the normalized potential $\Gamma$ (unitless) obtained though finite element simulations at a pore water conductivity of $\sigma_{w}=10 \mathrm{~S} \mathrm{~m}^{-1}$ and saturation $s_{w}$ $=0.9086$. The spheres shown in the pore space represents insulating gas bubbles. We consider that the gas bubbles are excluded from the dead-end and the electrical double layer. The electrical field remains strong in the throat.

(pore volume divided by the total volume) is 0.332 . The ratio $S / V_{p}$ determined from the microgeometry shown in Fig. $2 \mathrm{a}$ is $0.2704 \mathrm{~nm}^{-1}$. Therefore the hydraulic radius $V_{p} / S$ is on the order of $3.7 \mathrm{~nm}$. The solid phase is coated by a layer of thickness $x_{\mathrm{s}}=1 \mathrm{~nm}$ with a conductivity $\sigma_{s}$ of $1 \mathrm{~S} \mathrm{~m}^{-1}$ (therefore $\Sigma_{s}=10^{-9} \mathrm{~S}$ ).

The distribution of the normalized electrical potential $\Gamma$ is shown at high salinity in Fig. 3 and at low-salinity in Fig. 5. We observe that the distributions of the equipotentials are completely different in the two 


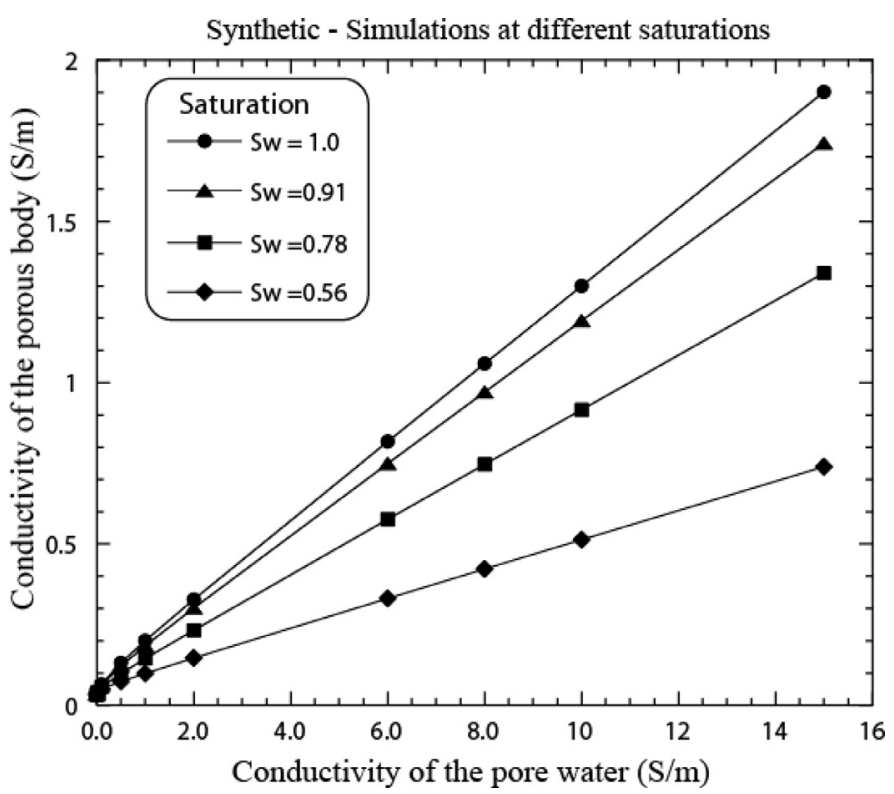

Fig. 9. Finite element simulations of the electrical conductivity of the porous material as a function of the pore water conductivity for different value of the pore water saturations. The lines are just guides for the eyes.

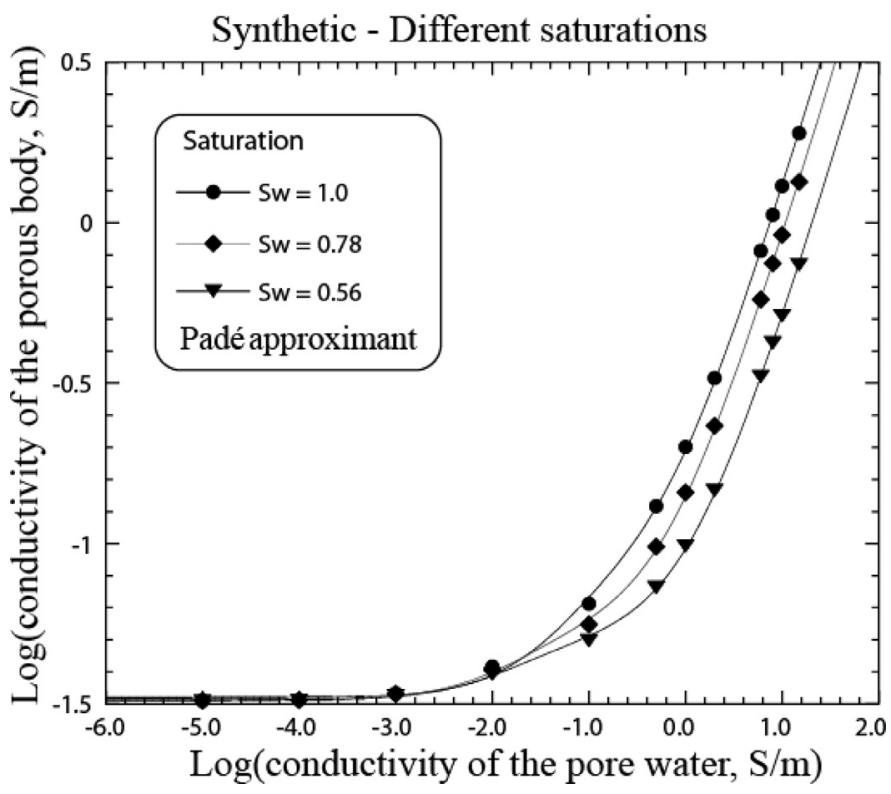

Fig. 10. Finite element simulations of the electrical conductivity of the porous material as a function of the pore water conductivity for different value of the pore water saturations. The lines correspond to the best fit of the Padé approximant.

cases. We see clearly that the conduction paths are vastly different at high and low salinities while in the model developed by Pride (1994), this is not the case indicating a serious flaw in his model. We performed the simulations for a set of 12 pore water conductivities $\sigma_{w}$ covering the high and low salinity ranges $\sigma_{w}=0,10^{-5}, 10^{-4}, 10^{-3}, 10^{-2}, 10^{-1}, 0.5$, $1,2,6,8$, and $10 \mathrm{~S} \mathrm{~m}^{-1}$ while $\sigma_{s}=1 \mathrm{~S} \mathrm{~m}^{-1}$ (constant).

From their definitions in terms of integral equations (see Eqs. (12), (19), (25), and (26)), we determined the values of the four petrophysical parameters of interest using the potential distributions $\Gamma_{b}$ and $\Gamma_{S}$ shown in Figs. 3 and 5. We obtain $F=5.0$ (from Eq. (12)) and therefore the bulk tortuosity is $\alpha_{b}=F \phi=1.7$. The pore size length $\Lambda$ is $4.9 \mathrm{~nm}$ (from Eq. (19)), the low-salinity formation factor is $f=31 \mathrm{~nm}$ (from Eq. (25)) and
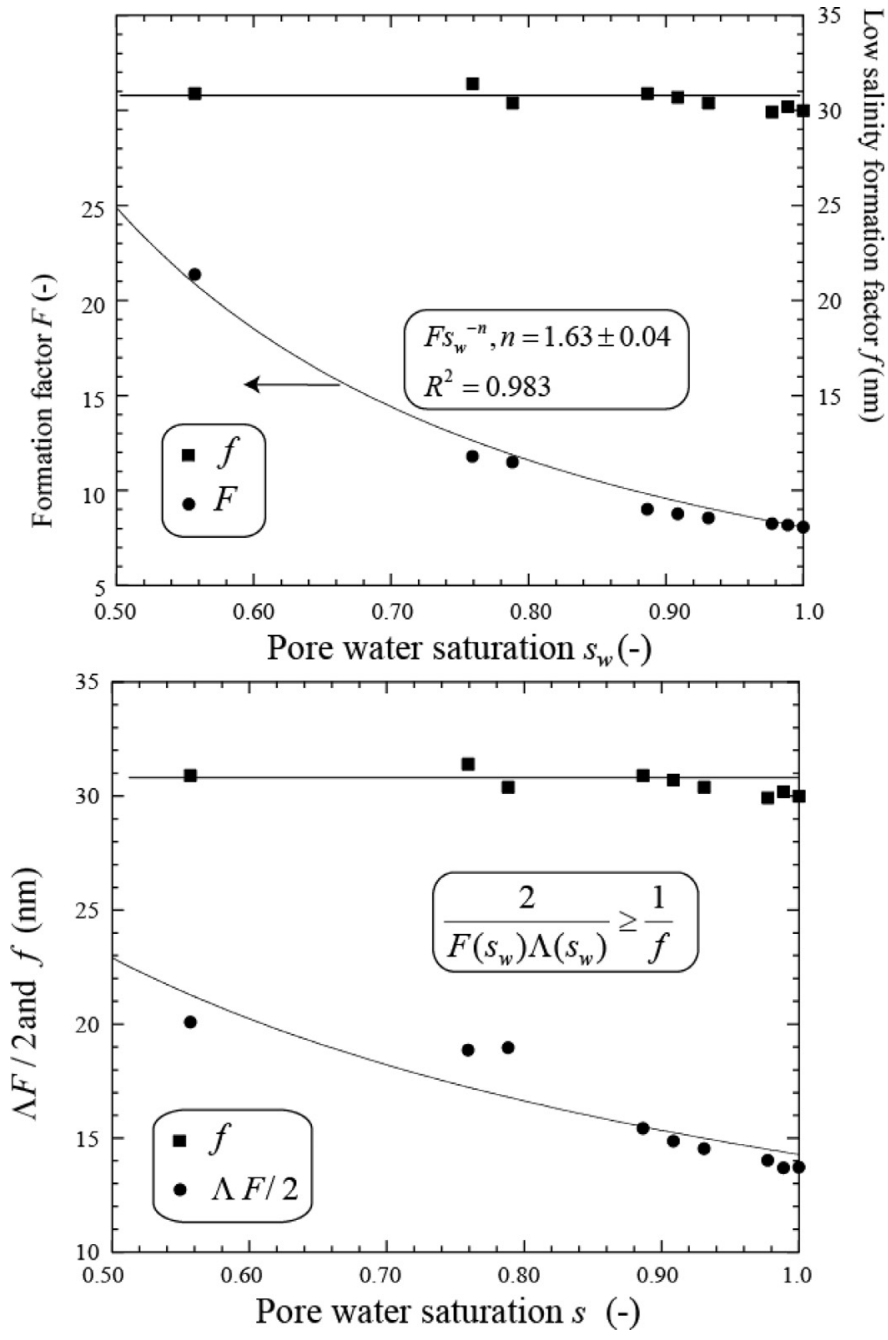

Fig. 11. High and low salinity formation factors ( $F$ and $f$ ) as a function of the water saturation for the synthetic model shown in Fig. 2. The simulations confirm that $f$ is independent of the saturation while $F$ can be replaced by a second Archie's law with a saturation exponent $n$. The lower figure allows checking that the property $2 /(F \Lambda) \geq 1 / f$ (the filled squares correspond to the low salinity formation factor) is always respected at all the saturations.

$\lambda=113 \mathrm{~nm}$ integrating over the whole pore space including the double layer with Eq. (26). This yields therefore a surface tortuosity $\alpha_{s}=2.8$, therefore higher (as expected) than the bulk tortuosity. The pore sizes $\Lambda$ is comparable to the pore size of the throat which is $4 \mathrm{~nm}$ from the geometry shown in Fig. 2. The cementation exponent $m$ in Archie's law $F=\phi^{-m}$ is 1.46 , which is obtained from $F$ and $\phi$ using $m=-\ln F / \ln \phi$. Note that we can check the validity of Eq. (67), $\Lambda=(2 / m) V_{p} / S$. Indeed, taking $m=1.46, V_{p} / S=3.7 \mathrm{~nm}$ we obtain $\Lambda=5.1 \mathrm{~nm}$ with Eq. (67), close to the value provided above $(4.9 \mathrm{~nm})$ from the integral Eq. (19).

The conductivity curve is shown in Figs. 6 and 7. This curve is clearly characterized by a high salinity asymptotic limit as predicted by the theory. From the slope and the $y$-intercept and using Eq. (40), the apparent formation factor is $F_{a}=8.4$ while the apparent effective pore size $\Lambda_{a}=$ $2.9 \mathrm{~nm}$. From Eq. (65), we have $\Lambda=\Lambda_{a}+2 x_{s}$ and therefore using this equation with $\Lambda_{a}=2.9 \mathrm{~nm}$ (from the data shown in Fig. 6), the value of $x_{\mathrm{s}}=1 \mathrm{~nm}$ (thickness of the electrical double layer), we obtain $\Lambda=$ $4.9 \mathrm{~nm}$. This value is consistent with the value determined above from the integral Eq. (19), showing again the self-consistency of the model proposed in this paper.

From this value of $\Lambda=4.9 \mathrm{~nm}$, we can compute the value of the formation factor $F$ using Eq. (64) i.e., $F=F_{a}\left(1-2 x_{s} / \Lambda\right)$. This yields $F$ 


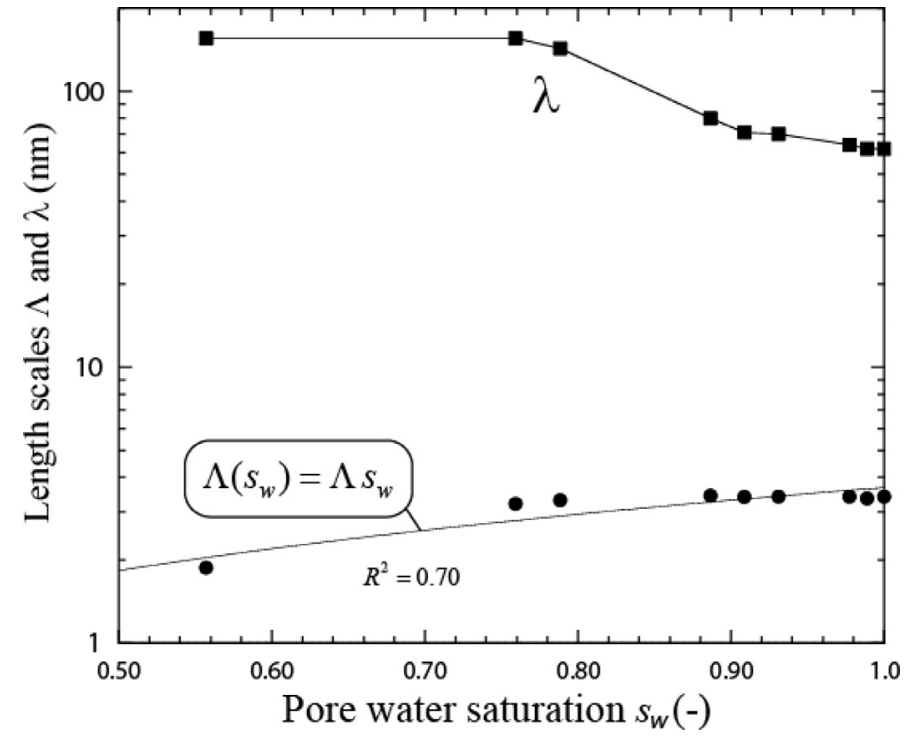

Fig. 12. High and low salinity characteristic length scales ( $\Lambda$ and $\lambda)$ as a function of the water saturation for the synthetic model shown in Fig. 2. The textural parameters are determined by fitting the Padé approximant to the synthetic data obtained by finite element modeling. The simulations confirm that $\Lambda$ scales approximately with the saturation while $\lambda$ is difficult to determine accurately and close to the value given at saturation from its integral form $(113 \mathrm{~nm})$. We also check the property $\lambda>\Lambda$.

$=5.0$. This value is again consistent with the values determined from the integral equation (Eq. (12), see discussion above). The same type of analysis can be done for the low salinity asymptotic limit. If we consider the case for which the conductivity of the pore water is zero, $\sigma=Q_{S} B / f$, we obtain $f=31 \mathrm{~nm}$. The slope of the trend at low salinities is 1.8018 (determined over the three last salinities). Since the slope is $\left(\lambda-2 x_{s}\right) / 2 f$, this yields $\lambda=113 \mathrm{~nm}$. Therefore the dual water correction is vanishingly small at low salinities. In Fig. 7, we also fit the data with the Padé approximant. We obtain $F_{a}=8.1$ (close to the value obtained with the

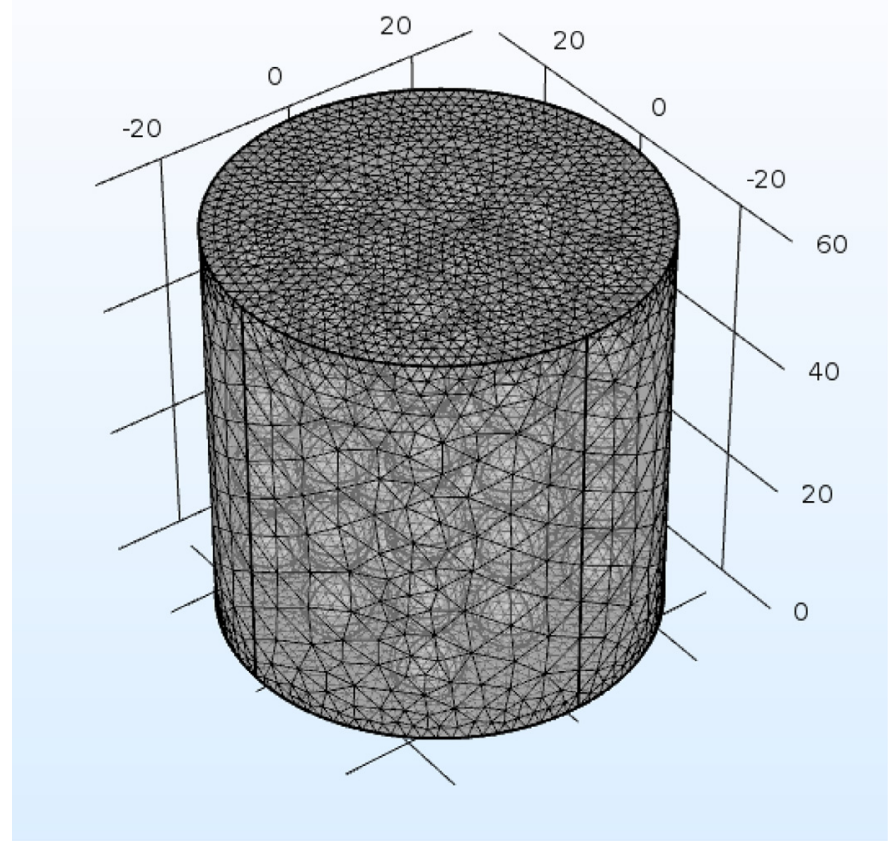

Fig. 13. Granular material idealized as sphere pack and finite-element mesh. The current is injected between the two end-faces of the cylinder while an insulating boundary condition is applied on the side boundary.

high salinity asymptotic limit), $\Lambda_{a}=3.4 \mathrm{~nm}$ (close to the value obtained with the high salinity asymptotic limit), $f=30 \mathrm{~nm}$ and $\lambda=62 \mathrm{~nm}$.

We perform now finite element modeling at partial saturations. These finite element simulations are performed by adding insulating spheres in the pore space to simulate the presence of gas bubbles. An example of the $\Gamma$-potential distribution is shown in Fig. 8. Simulations were performed at the following gas saturations: [0, 0.011, 0.023, 0.069, $0.091,0.113,0.217,0.241,0.443,0.656]$. Examples of conductivity curves at different saturations are shown in Figs. 9 (linear-linear space) and 10 (log space). The curves shown in Fig. 9 looks like the type of

\section{a. High salinity potential distribution}

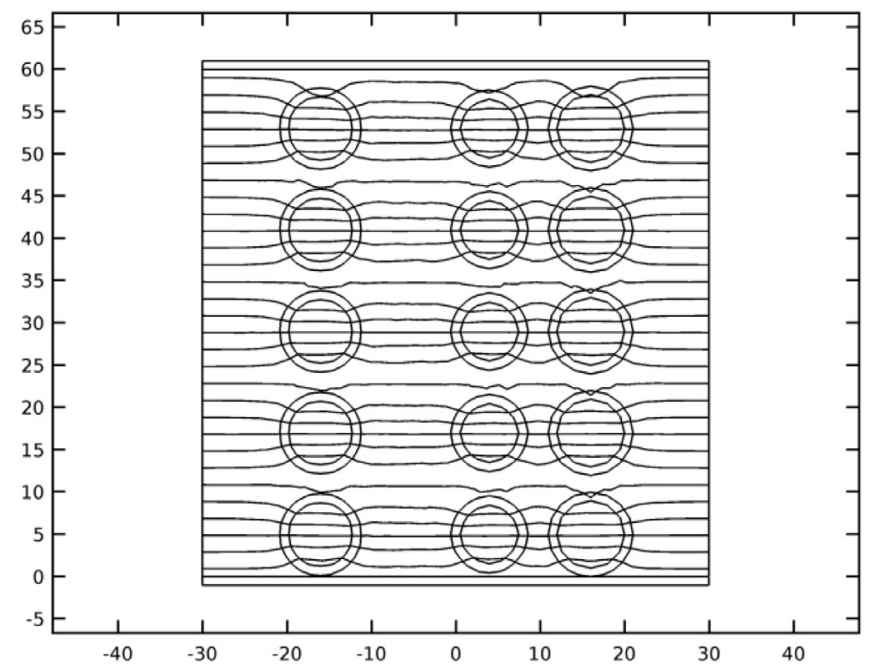

\section{b. Low salinity potential distribution}

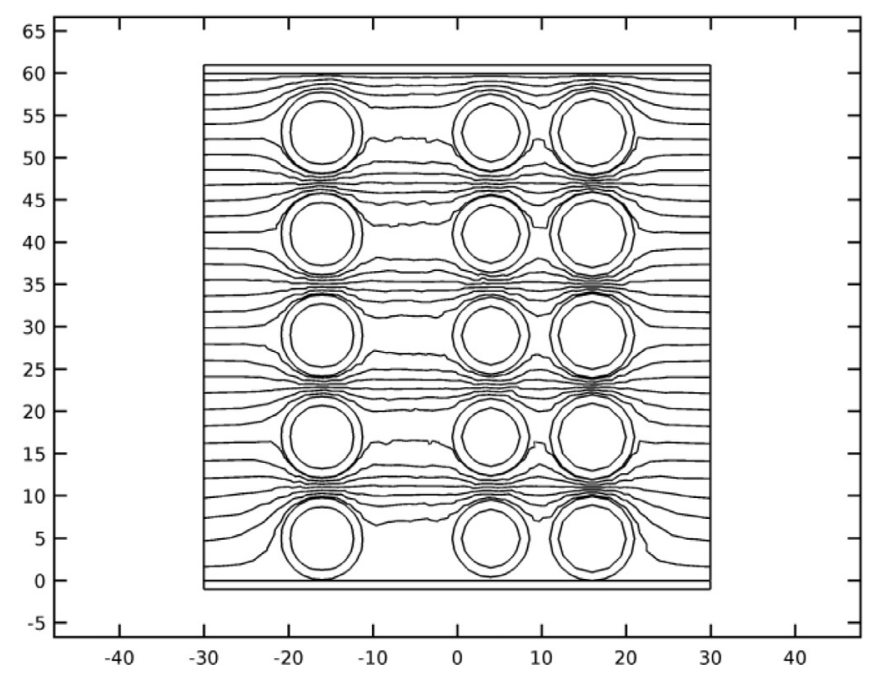

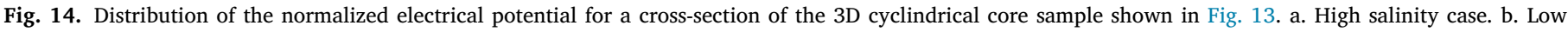

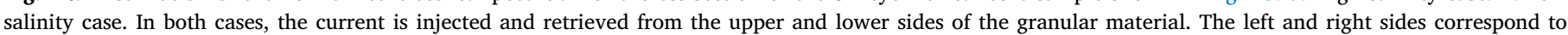

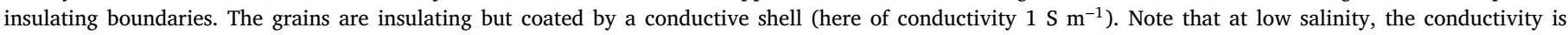
becoming independent of the conductivity of this shell. 


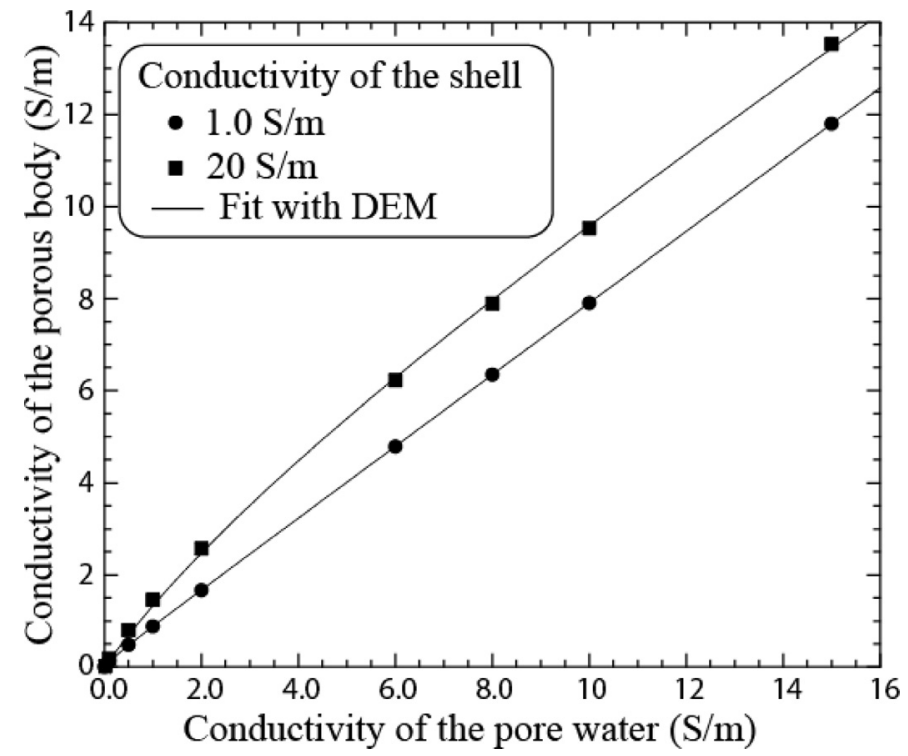

Fig. 15. Conductivity curves for the second synthetic case corresponding to a granular porous material with the insulating grains having a conductive shell of $1 \mathrm{~S} \mathrm{~m}^{-1}$ and $20 \mathrm{~S} \mathrm{~m}^{-1}$, respectively. The plain lines correspond to the fit obtained with the differential effective medium theory (DEM) using a least square fitting technique. In the first case (double layer conductivity of $1 \mathrm{~S} \mathrm{~m}^{-1}$ ), we obtain $F=1.285 \pm 0.001$ and $\sigma_{s s}=0.30 \pm 0.02 \mathrm{~S} \mathrm{~m}^{-1}\left(R^{2}=1.0\right)$. In the second case (double layer conductivity of $20 \mathrm{~S} \mathrm{~m}^{-1}$ ), we obtain $F=1.46 \pm 0.04$ and $\sigma_{s s}=7.8 \pm 0.3$ $\mathrm{S} \mathrm{m}^{-1}\left(R^{2}=0.9997\right)$.

curve for soils at partial saturations presented by Rhoades et al. (1989). Like in Fig. 7, we fit all the data using the Padé approximant Eqs. (36) and (37). The two formation factors $F$ and $f$ are plotted as a function of the saturation in Fig. 11 where we also demonstrate that the inequality $2 /(F \Lambda) \geq 1 / f$ (see Eq. (32) for the saturated case) is obeyed at all the saturations. The apparent formation factor scales with saturation according to the second Archie's law (see Eq. (95) with $n=1.63$ ). The formation factor $\phi$ is independent of the saturation as expected form its integral formulation.

In Fig. 12, we show how the two length scales $\lambda$ and $\Lambda$ change with the saturation (note that the inequality corresponding to Eq. (33)), i.e., $\lambda \geq \Lambda$ is also always respected). The length $\Lambda$ scales approximately with the saturation (see Eq. (13)) while $\lambda$ has a more complicated dependence with the saturation but its value is around $113 \mathrm{~nm}$, close to the value at saturation determined above.

The goal of the last numerical experiment is to numerically test the differential effective medium theory in fully saturated conditions (Fig. 13). We performed a finite element simulation for an assemblage of grains coated by an electrical double layer with an intrinsic conductivity of $1 \mathrm{~S} \mathrm{~m}^{-1}$ and $20 \mathrm{~S} \mathrm{~m}^{-1}$ (to observe the effect of the surface conductivity on the curvature). The pore water is changed using the following range of pore water conductivities $\left[10^{-4}, 10^{-3}, 10^{-2}, 10^{-1}, 0.5\right.$, $1.0,2.0,6.0,8,10$, and $\left.15 \mathrm{~S} \mathrm{~m}^{-1}\right]$. The dimension of the cylindrical synthetic sample is $30 \mu \mathrm{m}$ (radius) by $60 \mu \mathrm{m}$ (Fig. 13). The number of finite element mesh for the simulation is 152,032 (Fig. 13) and we check that the potential distributions obtained at high and low salinities (see Fig. 14) are mesh-independent. The grains are represented by spheres with a radius of $5 \mu \mathrm{m}$ while the thickness of the double layer is $1 \mu \mathrm{m}$. There are 55 grains in the simulation and the resulting porosity is 0.830 . We have $S / V_{p}=0.123 \mu \mathrm{m}^{-1}$ (using the surface of the 55 grains divided by the pore volume).

We start with the analysis of the two conductivity curves shown in Fig. 15. First the conductivity of the porous material goes to zero when the conductivity of the pore water goes to zero as expected. The differential effective medium theory is able to represent the conductivity curves obtained by solving Laplace equation. When the conductivity of
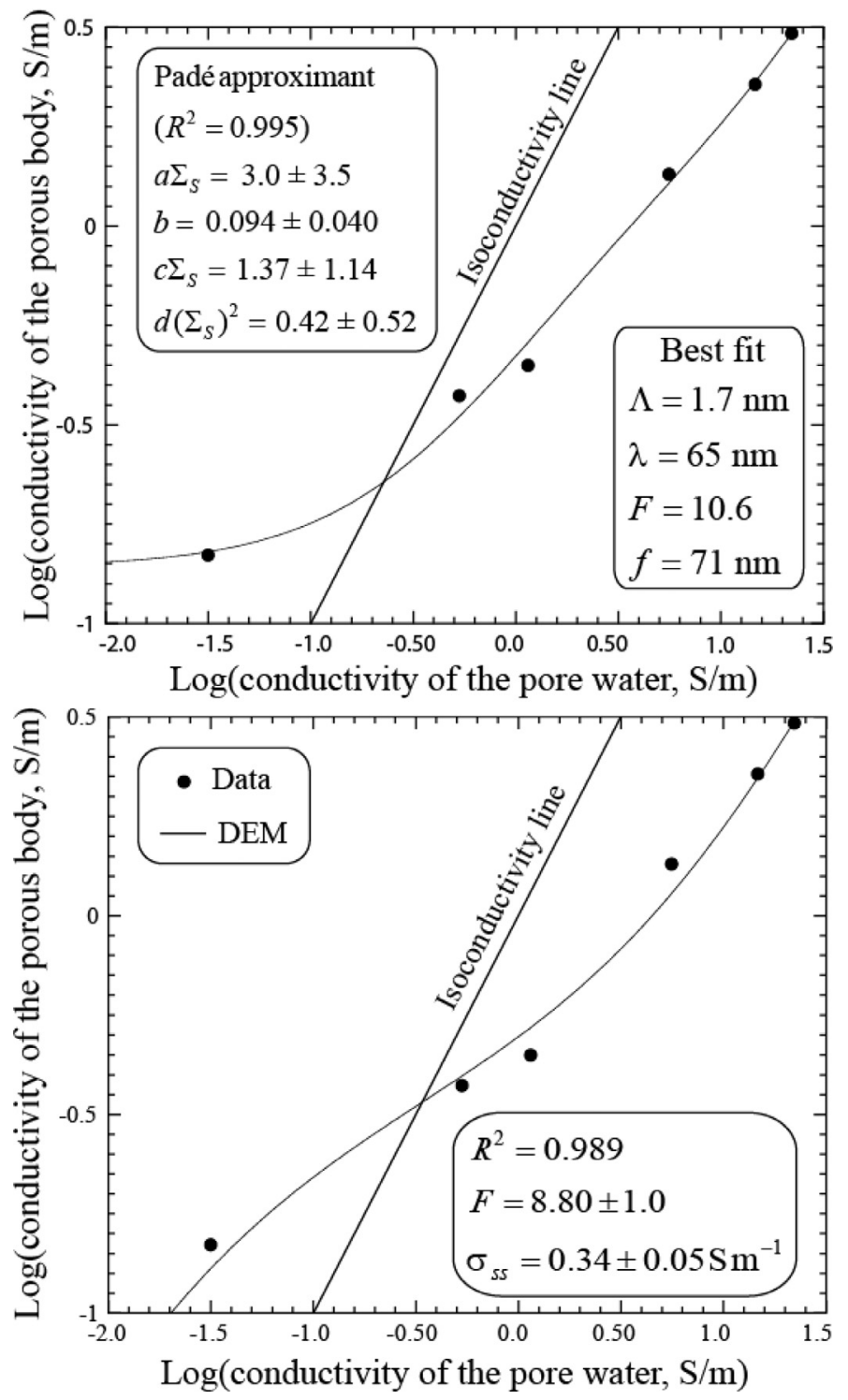

Fig. 16. Conductivity curve for a soil sample. a. Best fit in a log space of the Padé approximant used to represent the conductivity of a soil sample (sandy clay) as a function of the pore water conductivity. Three out of four textural parameters present strong uncertainties in their determination. We check that the properties $\lambda \geq \Lambda$ and $\lambda \geq 2 f / F$ are obeyed. b. Best fit in a log space of the analytical solution of the differential effective medium theory $\backslash$ used to represent the conductivity of a soil sample (sandy clay) as a function of the pore water conductivity. The property $\sigma_{s s}=\sigma_{S} / m$ with $\sigma_{S}=0.77 \mathrm{~S} \mathrm{~m}^{-1}$ yields $m=2.26$. Using a formation factor $F=8.80$ this yields a porosity of 0.35 . The measured porosity is $\phi=0.522$.

the shell is $1.0 \mathrm{~S} \mathrm{~m}^{-1}$, we obtain a formation factor of $F=1.285 \pm 0.001$ (therefore $m=1.35$ ) and a grain conductivity of $\sigma_{s s}=0.30 \pm 0.02 \mathrm{~S} \mathrm{~m}^{-1}$ $\left(R^{2}=1.0\right)$. At low pore water conductivity (not shown here) the two conductivity curves superimposes, which means that the conductivity of the porous material is independent of the conductivity of the grains. The explanation of this behavior is provided in Fig. 14 where it is shown that at low pore water salinities, the conduction paths avoid the grains.

\subsection{Laboratory experiments}

We now show how the equations discussed in the previous sections can be applied to real data. We first use a soil sample from the recent study of Revil et al. (2017b). This sample is a sandy clay (Sample AE). 

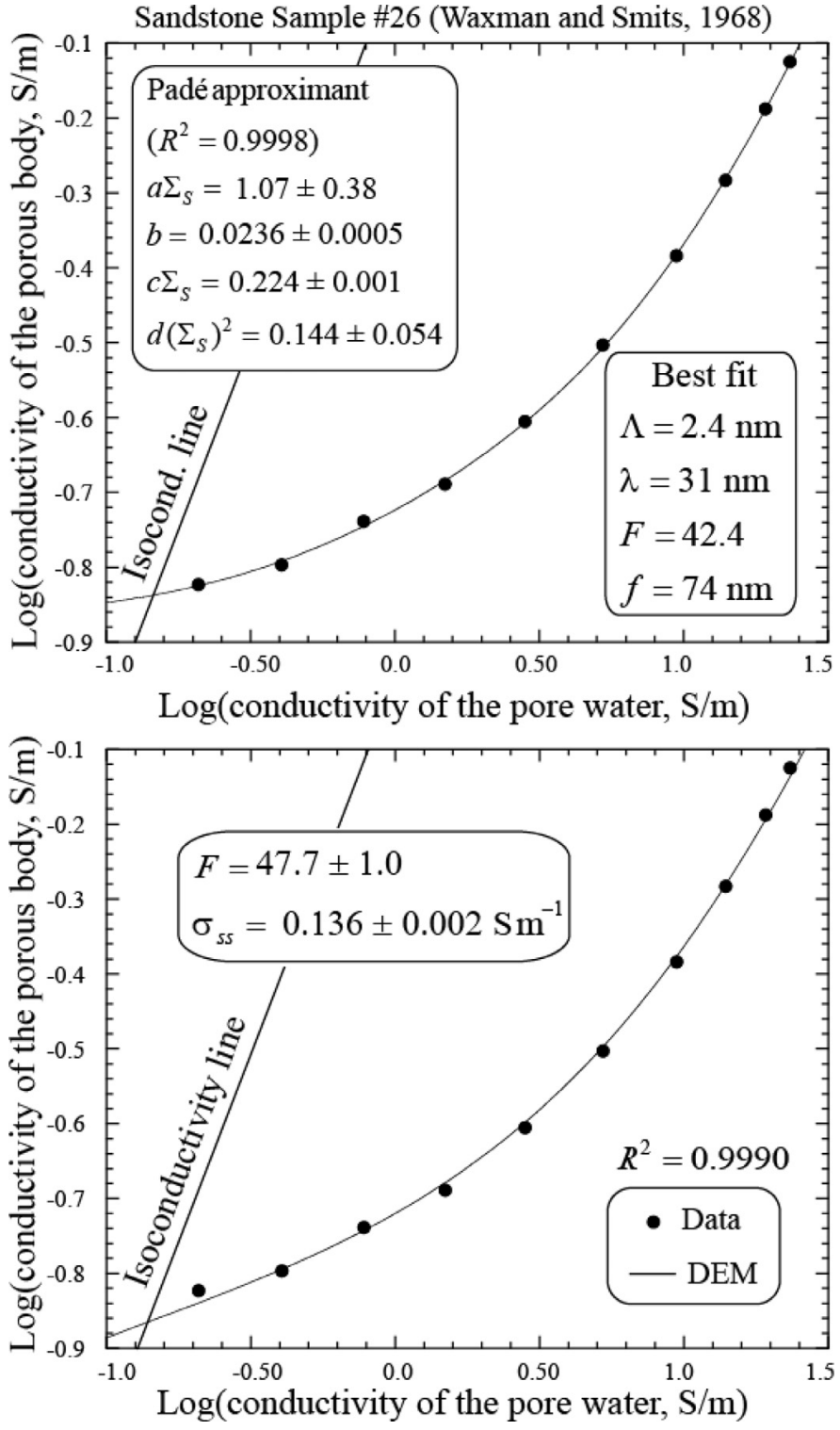

Fig. 17. Best fit in a log space of the Padé approximant and differential effective medium theory used to represent the conductivity of a shaly sand (sample \#26 from Waxman and Smits, 1968). The clay fraction of this sandstone is $100 \%$ Montmorillonite. Since its porosity is 0.229 , the cementation exponent $m$ is 2.54 . For the first case, we use a specific surface conductivity $\Sigma_{\mathrm{S}}=1 \times 10^{-8} \mathrm{~S}$. We check that $\lambda \geq \Lambda$ and $\lambda \geq 2 f / F$.

The conductivity curve is shown in a log-log space in Fig. 16. We use the Padé approximant to fit the data and we obtain $\Lambda=1.7 \mathrm{~nm}, F=10.6$, $f=71 \mathrm{~nm}$, and $\lambda=65 \mathrm{~nm}$. As we can see, some of the model parameters are poorly determined. We use a specific surface conductivity $\Sigma_{S}=$ $1 \times 10^{-8} \mathrm{~S}$ based on the study of Revil and Skold (2011). As expected for a granular material, the values of $f$ and $\lambda$ are very large with respect to $\Lambda$.

In Fig. 16, we also use the differential effective medium approach to fit the data. Like the Padé approximant, the model is able to fit the data very well but with two parameters only (the formation factor and the grain conductivity) instead of four parameters. This indicates the usefulness of the differential effective medium approach in capturing the non-linearity of the conductivity data. We have performed a linear fit of the high salinity data in a linear-linear space using Eq. (2) (fit not shown here). We obtain $F=9.75 \pm 0.10$ and $\sigma_{S}=0.77 \pm 0.02 \mathrm{~S} \mathrm{~m}^{-1}$ $\left(\mathrm{R}^{2}=0.9999\right)$. Using this surface conductivity, the length scale $\Lambda$ is given by $\Lambda=2 \Sigma_{S} /\left(F \sigma_{S}\right)=2.7 \mathrm{~nm}$ using $\Sigma_{S}=1 \times 10^{-8}$ S relatively consistent with the result derived in Fig. $16 \mathrm{a}(\Lambda=1.7 \mathrm{~nm})$.

The second sample we investigate is sample \#26 from the collection of Waxman and Smits (1968). This is a shaly sand with a porosity $\phi$ of 0.229 . We obtain $F=42.4$ and $\Lambda=2.4 \mathrm{~nm}$. The cementation exponent $m$ is therefore $m=-\ln F / \ln \phi=2.54$. Taking $\Lambda=(2 / m) V_{p} / S$ with $\Lambda=$ $2.4 \mathrm{~nm}$, we have $V_{p} / S=3.1 \mathrm{~nm}$ (and therefore with $V_{p} / S \phi=13.5 \mathrm{~nm}$, we can check that $f \geq V_{p} / S \phi$ ). The data are fitted in Fig. 17 with both the Padé approximant and the differential effective medium theory. Both models are able to fit the data but again the differential effective medium theory is able to fit the data with only two parameters, which makes it attractive for field applications in hydrogeophysics.

\section{Conclusions}

We have reviewed a conductivity model that can be derived from volume-averaging arguments and we have connected this model to various empirical and semi-empirical conductivity models broadly used in the literature. At very low salinities (i.e., for freshwater environments), it is important to capture the non-linearity of the conductivity curve to interpret electrical resistivity data in field conditions. The following conclusions have been reached.

(1) We have shown that the use of a Padé approximant can be used to build a non-linear conductivity model from rigorously defined asymptotic behaviors at low and high salinities. Furthermore, we have shown for the first time that this Padé-based model is equivalent to the non-linear empirical model developed by Wyllie and Southwick (1954), which assumes three electrical resistances forming a network. They are (1) a resistance consisting of grains in electrical contact with each other. (2) a resistance parallel to the previous one and consisting of the pore water located in the connected pore space, and (3) a resistance consisting of grains and pore water in series with each other. All the four textural coefficients entering this model are now perfectly identified with respect to four fundamental textural properties of porous bodies. The non-linearity is important for materials characterized by small pore sizes and high cation exchange capacities such as smectite-rich soils and bentonite.

(2) The dual water model is an empirical model initially proposed by Clavier et al. (1984) to emphasize the volume fractions occupied by the electrical double layer and free-pore water in the expression of the electrical conductivity of a porous material. The dual water model can be explained from the definition of the specific surface conductivity at the pore water / mineral interface, which should only accounts for the excess conductivity above the pore water conductivity. For low-porosity materials characterized by small sizes of their pore throats, this correction is important and cannot be neglected. This type of materials is also the one characterized by high CEC and strong non-linearity in the conductivity versus pore water conductivity curve.

(3) We have also connected the Padé-based conductivity model to the differential effective medium conductivity solution of a granular medium. For granular media, the differential effective medium model is probably the best model to capture the non-linear behavior with only two materials properties (instead of four for the Padébased conductivity model). The conductivity of the grains in this mode can be rigorously connected to the CEC.

(4) For all the conductivity models, the effect of saturation can be accounted for. Having data at various saturations can be important to get further constraints regarding the four textural parameters entering the general conductivity equation.

(5) A comparison between the Padé-based conductivity model and numerical simulations allows to better understand the characteristics of the electrical conductivity curve and to evaluate the different approximations made in the various models. The Padé-based conductivity model and the differential effective medium-based model can 
replicate the non-linear behavior of the conductivity curve observed in both the numerical models and in the experimental data.

\section{Acknowledgments}

This contribution is supported by the University of Melbourne through a project funded by the Commonwealth of Australia (contract CR-2016-UNIV.MELBOURNE-147672-UMR5275). The numerical data and all the data used to generate the figures can be obtained by sending an email to the corresponding author (A. Revil, andre.revil@univsmb.fr). They will be provided on ResearchGate upon the publication of the manuscript.

\section{Supplementary materials}

Supplementary material associated with this article can be found, in the online version, at doi:10.1016/j.advwatres.2018.08.007.

\section{Appendix A. Surface and bulk tortuosities}

We have already introduced two tortuosities, one for the bulk pore space at high salinities and one at low salinity for conduction in the electrical double layer. We investigate the inequality between these two tortuosities. We start with the inequality,

$$
\frac{2}{F \Lambda} \geq \frac{1}{f}
$$

$F \leq \frac{2 f}{\Lambda}$,

$\frac{\alpha_{b}}{\phi} \leq \frac{2 f}{\Lambda}$

where we have used the relationship between the bulk tortuosity and the formation factor $F=\alpha_{b} / \phi$. In addition, we have $2 / \Lambda \approx m S / V_{p}$. This yields,

$\frac{\alpha_{b}}{\phi} \leq m f \frac{S}{V_{p}}$.

Finally the surface tortuosity is defined as $\alpha_{S}=f \phi S / V_{p}$. This leads to

$\alpha_{b} \leq m \alpha_{S}$

\section{Appendix B. Asymptotic limits}

Using the expression of the Padé approximant, we have

$\lim _{\frac{2}{\Lambda} x \ll 1} g(x)=b+(c-a b) x+O\left(\frac{2}{\Lambda} x\right)^{2}$,

$\lim _{\frac{2}{\lambda} x \gg 1} g(x)=\frac{c}{a}+\frac{d}{a} x+O\left(\frac{\lambda}{2} \frac{1}{x}\right)^{2}$,

which can be compared to Eqs. (38) and (39). Using the identifications, we obtain

$b=\frac{1}{F}$,

$c-a b=\frac{2}{F \Lambda}$,

$\frac{c}{a}=\frac{\lambda}{2 f}$,

$\frac{d}{a}=\frac{1}{f}$

In turn this yields the expressions obtained in the main text.

\section{Appendix C. Differential effective medium theory}

The solution of the upscaling procedure using the differential effective medium theory can be written as (e.g., Sen et al., 1981),

$\frac{\sigma-\sigma_{s s}}{\sigma_{w}-\sigma_{s s}}\left(\frac{\sigma_{w}}{\sigma}\right)^{D}=\phi$,

where $D=1-1 / m$ is called the depolarization factor (e.g., Bussian, 1983). Eq. (C1) can easily be written as,

$\sigma=\sigma_{w} \phi^{\frac{m}{1-m}}\left(\frac{1-\sigma_{w} / \sigma_{s s}}{1-\sigma / \sigma_{s s}}\right)^{\frac{m}{1-m}}$,

which is used in the main text to define the low salinity asymptotic limit of the conductivity equation for granular materials. To find the other formula, we need to proceed as follows. First we rewrite Eq. (C1) as,

$\left(\frac{\sigma}{\sigma_{w}}\right)\left(\frac{1-\sigma_{s s} / \sigma}{1-\sigma_{s s} / \sigma_{w}}\right)\left(\frac{\sigma_{w}}{\sigma}\right)^{D}=\phi$,

$\left(\frac{1-\sigma_{s s} / \sigma}{1-\sigma_{s s} / \sigma_{w}}\right)\left(\frac{\sigma_{w}}{\sigma}\right)^{D-1}=\phi$

with $D-1=-1 / m$. In turn this yields,

$\sigma=\sigma_{w} \phi^{m}\left(\frac{1-\sigma_{s s} / \sigma_{w}}{1-\sigma_{s s} / \sigma}\right)^{m}$.

Eqs. (C2) and (C4) are therefore equivalent.

\section{References}

Abuel-Naga, H.M., Bouazza, A., February 2016. Electrical resistance method for assessing spatial variation of water content in geosynthetics clay liners at laboratory scale. Geotext. Geomemb. 44 (1), 117-121. https://doi.org/10.1016/j.geotexmem.2015.07.002.

Al Hagrey, S.A., 2012. 2D optimized electrode arrays for borehole resistivity tomography and $\mathrm{CO}_{2}$ sequestration modelling. Pure Appl. Geophys. 169 (7), 1283-1292.

Archie, G.E., 1942. The electrical resistivity $\log$ as an aid in determining some reservoir characteristics. Trans. AIME 146, 54-62.

Bernabé, Y., Revil, A., 1995. Pore-scale heterogeneity, energy dissipation and the transport properties of rocks. Geophys. Res. Lett. 22 (12), 1529-1552.

Binley, A., Hubbard, S.S., Huisman, J.A., Revil, A., Robinson, D.A., Singha, K., Slater, L.D., 2015. The emergence of hydrogeophysics for improved understanding of subsurface processes over multiple scales. Water Resources Res. 51, 3837-3866. https://doi.org/10.1002/2015WR017016.

Börner, J.H., V., Herdegen, J.-U., Repke, K., Spitzer, 2013. The impact of $\mathrm{CO}_{2}$ on the electrical properties of water bearing porous media - laboratory experiments with respect to carbon capture and storage. Geophys. Prospect. 61, 446-460.

Bruggeman, D.A.G., 1935. The calculation of various physical constants of heterogeneous substances. I. The dielectric constants and conductivities of mixtures composed of isotropic substances. Ann. Physik. 24, 636-664.

Bussian, A.E., 1983. Electrical conductance in a porous medium. Geophysics 48 (9), 1258-1268.

Clavier, C., Coates, G., Dumanoir, J., 1984. The theoretical and experimental bases for the "Dual Water" Model for the interpretation of shaly sands. Soc. Petrol. Eng. J. 24 (2), 153-168. https://doi.org/10.2118/6859-PA.

Cremers, A., Laudelout, H., 1965. On the "isoconductivity value" of clay gels. Soil Sci. 100, 298-299.

Cremers, A., Van Loon, J., Laudelout, H., 1966. Geometry effects for specific electrical conductance in clays and soils. In: Proceedings of the 14th National Conference on Clays and Clay Minerals, 149, p. 162.

Day-Lewis, F.D., N., Linde, R., Haggerty, K., Singha, Briggs, M.A., 2017. Pore network modeling of the electrical signature of solute transport in dual-domain media. Geophys. Res. Lett. 44. https://doi.org/10.1002/2017GL073326.

Friedman, S.P., 2005. Soil properties influencing apparent electrical conductivity: a review. Comput. Electron. Agricult. 46, 45-70.

Greve, A.K., Roshan, H., B.F.J., Kelly, 2013. Electrical conductivity of partially saturated porous media containing clay: an improved formulation. J. Geophys. Res. 118, 3297-3303.

Hanai, T., 1960a. Theory of the dielectric dispersion due to the interfacial polarization and its application to emulsions. Kolloid-Zeitschrift 171, 23-31.

Hanai, T., 1960b. a remark on the "Theory of the dielectric dispersion due to the interfacial polarization:. Kolloid-Zeitschrift 175, 61-62.

Jackson, P.D., D.T., Smith, P.N., Stanford, 1978. Resistivity-porosity-particle shape relationships for marine sands. Geophysics 43, 1250-1268.

Johnson, D.L., Plona, T.J., H., Kojima, 1986. Probing porous media with 1st sound, 2nd sound, 4th sound and 3rd sound. In: Jayanth, R., Banavar, J., Winkler, K.W. (Eds.), Physics and Chemistry of Porous Media, 2,. American Institute of Physics, New York, pp. 243-277. Conf. Proc. No 154. 
Johnson, D.L., P.N., Sen, 1988. Dependence of the conductivity of a porous medium on electrolytic conductivity. Phys. Rev. B 37, 3502-3510.

Lima, A.L., Sharma, M., 1990. A grain conductivity approach to shaly sandstones. Geophysics 55 (10), 1347-1356.

Lima, A.T., Loch, J.P.G., Kleingeld, P.J., 2010. Bentonite electrical conductivity: a model based on series-parallel transport. J. Appl. Electrochem. 40, 1061-1068. https://doi.org/10.1007/s10800-009-0060-7.

Mualem, Y., S.P., Friedman, 1991. Theoretical prediction of electrical conductivity in saturated and unsaturated soil. Water Resources Res. 27, 2771-2777.

Nadler, A., 1982. Estimating the soil water dependence of the electrical conductivity soil solution/electrical conductivity bulk soil ratio. Soil Sci. Soc. Am. J. 46, 722-726.

Nadler, A., 1991. Effect of soil structure on bulk soil electrical conductivity (ECa) using the TDR and 4P techniques. Soil Sci 152, 199-203.

Patnode, H.W., Wyllie, J., 1950. The presence of conductive solids in reservoir rocks as a factor in electric log interpretation. Trans. A.I.M.E. i89, 47-52.

Pride, S.R., 1994. Governing equations for the coupled electromagnetics and acoustics of porous media. Phys. Rev. B 50, 15678-15696. https://doi.org/10.1103/PhysRevB.50.15678.

Priegnitz, M., Thaler, J., Spangenberg, E., Schicks, J.M., Schrotter, J., Abendroth, S., 2015. Characterizing electrical properties and permeability changes of hydrate bearing sediments using ERT data. Geophys. J. Int. 202, 1599-1612. https://doi.org/10.1093/gji/ggv245.

Revil, A., P.W.J., Glover, 1997. Theory of ionic surface electrical conduction in porous media. Phys. Rev. B 55 (3), 1757-1773. https://doi.org/10.1103/PhysRevB.55.1757.

Revil, A., L.M., Cathles, S., Losh, J.A., Nunn, 1998. Electrical conductivity in shaly sands with geophysical applications. J. Geophys. Res. 103 (B10), 23925-23936. https://doi.org/10.1029/98JB02125.

Revil, A., L.M., Cathles, 1999. Permeability of shaly sands. Water Resources Res. 35, 651662. https://doi.org/10.1029/98WR02700, 31999.

Revil, A., 2000. Thermal conductivity of unconsolidated sediments with geophysical applications. J. Geophys. Res. 105, 16749-16768.

Revil, A., Florsch, N., 2010. Determination of permeability from spectral induced polarization data in granular media. Geophys. J. Int. 181, 1480-1498. https://doi.org/10.1111/j.1365-246X.2010.04573.x, 2010.

Revil, A., Skold, M., 2011. Salinity dependence of spectral induced polarization in sands and sandstones. Geophys. J. Int. 187, 813-824. https://doi.org/10.1111/j.1365-246X.2011.05181.x.

Revil, A., Kessouri, P., Torres-Verdín, C., 2014. Electrical conductivity, induced polarization, and permeability of the Fontainebleau sandstone. Geophysics 79, 5, D301-D318. https://doi.org/10.1190/GEO2014-0036.1.

Revil, A., 2017a. Transport of water and ions in partially water-saturated porous media. Part 1. Constitutive equations. Adv. Water Resources 103, 119-138. https://doi.org/10.1016/j.advwatres.2016.02.006.

Revil, A., 2017b. Transport of water and ions in partially water-saturated porous media. Part 2. Filtration effects. Adv. Water Resources 103, 139-152. https://doi.org/10.1016/j.advwatres.2016.07.016.

Revil, A., Coperey, A., Shao, Z., Florsch, N., Fabricius, I.L., Deng, Y., Delsman, J.R, Pauw, P.S., Karaoulis, M., de Louw, P.G.B., van Baaren, E.S., Dabekaussen, W., Menkovic, A., Gunnink, J.L., 2017c. Complex conductivity of soils. Water Resour. Res. 53. https://doi.org/10.1002/2017WR020655.
Rhoades, J.D., P.A.C., Raats, R.J., Prather, 1976. Effects of liquid-phase electrical conductivity, water content, and surface conductivity on bulk soil electrical conductivity. Soil Sci. Soc. Am. J. 40, 651-655.

Rhoades, J.D., N.A., Manteghi, P.J., Shouse, W.J., Alves, 1989. Soil electrical conductivity and soil salinity: new formulations and calibrations. Soil Sci. Soc. Am. J. 53, 433-439.

Salem, H.S., Chilingarian, G.V., 1999. The cementation factor of Archie's equation for shaly sandstone reservoirs. J. Petrol. Sci. Eng. 23, 83-93.

Sauer Jr., M.C., Southwick, P.F., Spiegler, K.S., Wyllie, M.R.J., 1955. Electrical conductance of porous plugs. Ind. Eng. Chem. 47, 2187-2193.

Schwartz, L.M., Sen, P.N., Johnson, D.L., 1989a. Novel geometrical effects in electrolytic conduction in porous media. Physica A 157, 493-496.

Schwartz, L.M., Sen, P.N., Johnson, D.L., 1989b. Influence of rough surfaces on electrolytic conduction in porous media. Phys. Rev. B 40, 2450-2458 4.

Sen, P.N., C., Scala, M.H., Cohen, 1981. A self-similar model for sedimentary rocks with application to the dielectric constant of fused glass beads. Geophysics 46, 781-795.

Shabro, V., S.Kelly, C.Torres-Verdín, Sepehrnoori, K., Revil, A., 2014. Pore-scale modeling of electrical resistivity and permeability in FIB-SEM images of organic mudrock. Geophysics 79 (5), D289-D299. https://doi.org/10.1190/GEO2014-0141.1.

Shainberg, I., Levy, R., 1975. Electrical conductivity of Na-Monmorillonite suspensions. Clays Clay Miner. 23, 205-210.

Shainberg, I., J.D., Rhoades, R.J., Prather, 1980. Effect of ESP, cation exchange capacity, and soil solution concentration on soil electrical conductivity. Soil Sci. Soc. Am. J. 44, 469-473.

Spangenberg, E., 2001. Modeling of the influence of gas hydrate content on the electrical properties of porous sediments. J. Geophys. Res. 106 (B4), 6535-6548.

Street, N., 1963. On "the Isoconductivity value" of clays. Soil Sci. 95, 367.

Torskaya, T., Shabro, V., Torres-Verdín, C., Salazar-Tio, R., Revil, A., 2014. Grain shape shape effects on permeability, formation factor, and capillary pressure curve from pore-scale modeling. Transp. Porous Med. 102, 71-86. https://doi.org/10.1007/s11242-013-0262-7.

van Olphen, H., 1957. Surface conductance of various ion forms of bentonite in water and the electrical double layer. J. Phys. Chem 61, 1276-1280.

van Olphen, H., Waxman, M.H., 1958. Surface conductance of sodium bentonite in water: Proc. 5th Nat. Cotf Clays Clay Miner. 61-80.

Waxman, M.H., Smits, L.J.M., 1968. Electrical conductivities in oil bearing shaly sands. SPE Journal 8, 107-122.

Winsauer, W.O., 1952. Resistivity of brine-saturated sands in relation to pore geometry. AAPG Bull 36 (2), 253-277.

Wyllie., R.J., Southwick, P.F., 1954. An experimental investigation on the S.P. and resistivity phenomena in dirty sands. Trans. A.I.M.E. 201, 43-56.

Wyllie, M.R.J., Rose, W.D., 1950. Some theoretical considerations related to the quantitative evaluation of the physical characteristics of reservoir rock from electrical log data. J. Petrol. Tech. 2 (4), 105.

Wyllie, M.R.J., Southwick, P.F., 1954. An experimental investigation of the S. P. and resistivity phenomena in dirty sands. J. Petr. Tech. 6, 44-57. 